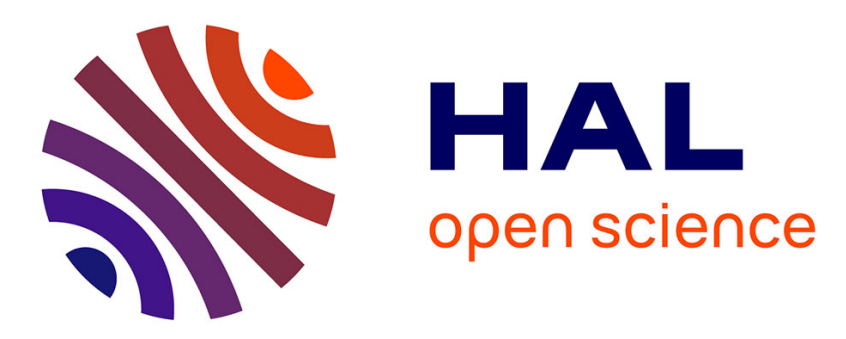

\title{
Evaluation of a charge-coupled-device-based video sensor for aircraft cargo surveillance
}

Thierry Sentenac, Yannick Le Maoult, Jean-José Orteu, G Boucourt

\section{To cite this version:}

Thierry Sentenac, Yannick Le Maoult, Jean-José Orteu, G Boucourt. Evaluation of a charge-coupleddevice-based video sensor for aircraft cargo surveillance. Optical Engineering, 2002, 41 (4), pp.796-810. 10.1117/1.1459450 . hal-01644901

\section{HAL Id: hal-01644901 \\ https://hal.science/hal-01644901}

Submitted on 6 Mar 2018

HAL is a multi-disciplinary open access archive for the deposit and dissemination of scientific research documents, whether they are published or not. The documents may come from teaching and research institutions in France or abroad, or from public or private research centers.
L'archive ouverte pluridisciplinaire HAL, est destinée au dépôt et à la diffusion de documents scientifiques de niveau recherche, publiés ou non, émanant des établissements d'enseignement et de recherche français ou étrangers, des laboratoires publics ou privés. 


\section{Evaluation of a charge-coupled-device-based video sensor for aircraft cargo surveillance}

\author{
Thierry Sentenac \\ Yannick Le Maoult \\ Jean-José Orteu \\ Ecole des Mines d'Albi-Carmaux \\ Route de Teillet \\ F-81013 Albi CT Cedex 09 \\ France \\ E-mail: sentenac@enstimac.fr \\ Gérard Boucourt \\ Latécoère \\ 35 rue de Périole \\ BP 5211, F-31079 Toulouse Cedex 5 \\ France
}

\begin{abstract}
We present a new video sensor for multimeasurements in an aircraft cargo compartment called a video sensor unit (VSU). This sensor, based on CCD technology, operates in the near IR (NIR) spectral band to measure temperatures due to overheating and fire events. After a characterization of the measurement chain, a radiometric model is applied to the VSU, enabling the measurement of temperatures ranging from 350 to $900^{\circ} \mathrm{C}$ with a noise equivalent temperature difference (NETD) lower than $\pm 8^{\circ} \mathrm{C}$. In the same spectral band (NIR), the VSU can also detect smoke and 3-D load displacements. The geometric model of the VSU is described and the associated calibration procedure is presented.
\end{abstract}

Subject terms: surveillance; cargo compartment; aircraft; charge-coupled-device camera calibration; radiometric model; geometric model; spatial resolution.

\section{Introduction}

The work presented in this paper is part of the Load Video Surveillance System (LVSS) project. The overall objective of this project is to develop a new approach to surveillance systems in aircraft cargo compartments based on imaging systems. The system is designed to measure two kinds of phenomena: load displacements and the appearance of fire from smoldering fires to flame and smoke.

The surveillance of aircraft cargo areas is usually limited to fire detection systems based on smoke sensors. According to the Federal Aviation Administration, ${ }^{1}$ for commercial aircraft, the rate of false alarms is estimated to be as high as 500:1. It has been suggested that instead of a local smoke concentration measurement, a multiparameter analysis (hot surfaces and/or flame together with smoke parameters) can improve the detection and greatly reduce the number of false alarms. In addition, some class cargo compartments are inaccessible during a flight. To ascertain the occurrence of a real fire, some researchers (see Refs. 2 and 3) have suggested monitoring the cargo area with imaging systems and coupling them with conventional sensors to increase the number of detection parameters. These systems are based on 3- to 5- $\mu \mathrm{m}$ IR or IR CCD cameras, which are often limited by their lack of robustness, high cost, and low spatial resolution. Microbolometer cameras have also proven to be interesting for use in airborne equipment, but there are only two suppliers and high spatial noise limits their performance for the moment.

In summary, our problem is to measure both load displacements and the parameters of fire appearance (temperature, surface, frequency) (Ref. 4) together with smoke $e^{5}$ with only one type of imaging sensor for cost saving. Instead of using conventional IR cameras, low-cost CCD cameras operating in the near IR (NIR) spectral band $(0.75$ to $1.1 \mu \mathrm{m})$ can be used. ${ }^{6-9}$ To meet the requirements of airborne systems, ${ }^{10}$ the CCD sensor, called a video sensor unit ${ }^{11}$ (VSU), consists of a protective glass window, a filter that selects the NIR spectral band, an optical system, a CCD detector, and some internal electronics. In addition, an active IR source is mounted inside the VSU to illuminate the cargo area. A photograph of the whole sensor is shown in Fig. 1.

The system was designed as follows to perform different detections. Smoldering fires and flame situations are characterized by mainly measuring their temperature. Next, the load displacement measurement is performed from a 3-D localization of the load between two successive image acquisitions. With monocular vision, the load localization can be computed only if the geometric model of the load and of the imaging sensor are determined. Note that the detection and the localization of each load is simplified by the use of a known specific target attached to each one and illuminated by the IR source (see Fig. 2). Finally, smoke detection is achieved by measuring its concentration in the same spectral band (given that the filter of the NIR spectral band selection cannot be removed). This result can be obtained from the measurement of the intensity variation of the illuminated targets (or additional ones) acting as calibrated reflectors. We must also be able to discriminate smoke from fog in nonpressurized aircraft cargo compartments.

The main purpose of this paper is to describe the design of the VSU based solely on CCD technology operating in the NIR spectral band. The VSU cannot only measure multiparameters but can also visually monitor the cargo compartment. The image analysis algorithms developed to detect load displacement, smoldering fires, flame, and smoke will be presented in a forthcoming paper.

The paper is organized as follows: Section 2 presents the characterization of the measurement chain from the scene intensity (hot sources and illuminated targets) to the expression of the VSU digital output signal via the influence of 


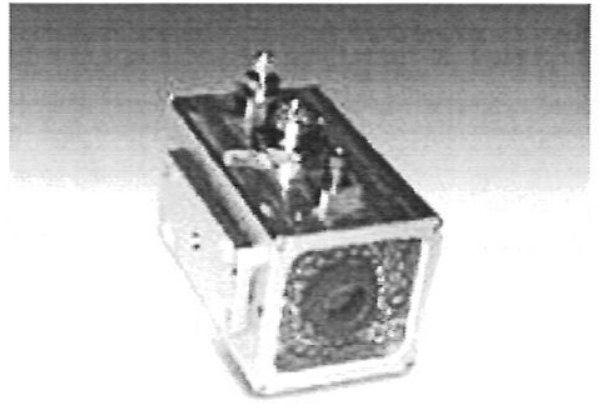

Fig. 1 VSU (Latécoére).

fog in the intensity of transmission. We also examine the problem of combining fire temperature and displacement measurements, which requires finding a trade-off in the VSU configuration to improve the output signal with respect to the requirement of spatial resolution. Based on this configuration, Sec. 3 provides the radiometric model of the VSU, which gives the effective relation between the temperature of the scene and the VSU digital output. Following the same idea, Sec. 3 also presents a geometric model enabling the measurement of the load movement based on the 3-D localization of targets within the cargo area. Section 3 also describes the calibration procedures for low-cost CCD devices that could potentially equip the VSU. Finally, Sec. 4 is devoted to temperature measurement examples completed by an analysis of temperature uncertainty.

\section{Characterization of the Measurement Chain}

The main objective of this section is to establish the ability of the VSU to analyze the scene intensity above all in smoldering fires or flame situations to measure temperatures with respect to the intensity from illuminated targets. Adding the spatial resolution requirements, this section determines the sensor configuration that enables both measurements. For one, the aim is to measure temperatures from 350 to $1000^{\circ} \mathrm{C}$ with a tolerance of $\pm 25^{\circ} \mathrm{C}$. For another, the minimal spatial displacement is $50 \mathrm{~mm}$ at a maximal observation distance of $15 \mathrm{~m}$. The measurement feasibility depends on the intensity of the scene, the environment

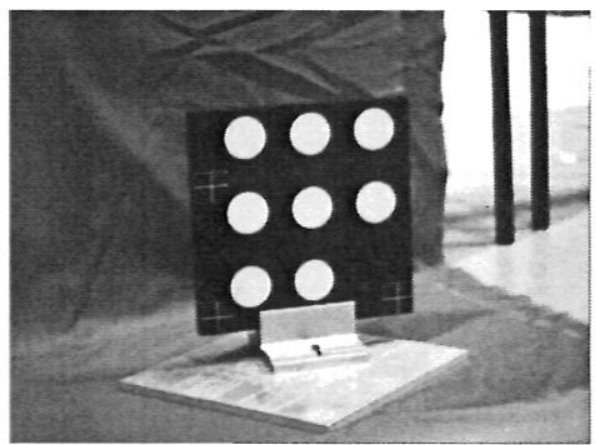

Fig. 2 Target for load displacement measurements

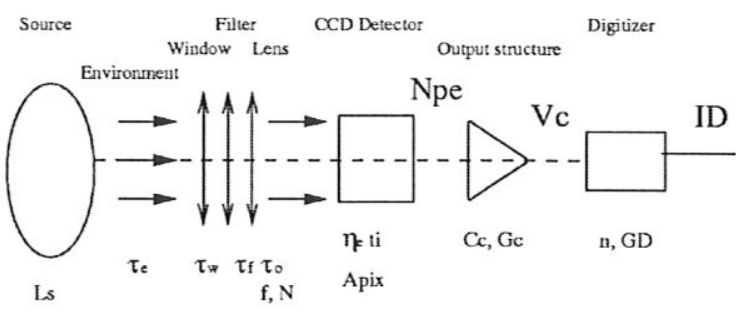

Fig. 3 Measurement chain (parameters are explained in text).

transmittance, and the sensitivity of the CCD detector. We set the values of the VSU parameters (see Fig. 3) to obtain a maximal output signal $I_{D}$ and to satisfy the spatial resolution requirements.

\subsection{Intensity of the Scene Sources $\left(L_{s}\right)$}

The scene intensity $L_{s}$ of hot sources, whose absolute temperature is $T$ (in degrees Kelvin) and whose spectral emissivity is $\varepsilon_{\lambda}$, is a spectral photon intensity $L_{\mathrm{sp}}$ described by Planck's radiation law as follows ${ }^{12}$ :

$L_{\mathrm{sp}}(\lambda, T)=\varepsilon_{\lambda} \frac{C_{3} \lambda^{-4}}{\exp \left(C_{2} / \lambda T\right)-1}\left(\right.$ photons $\left./ \mathrm{s} \mathrm{m}^{2} \mathrm{sr} \mu \mathrm{m}\right)$,

where $C_{2}$ and $C_{3}$ are the second and the third radiation constants $\left(C_{2}=(h c / k)\right.$ and $\left.C_{3}=2 c\right), h$ is Planck's constant $\left(h=6.62 \times 10^{-34} \mathrm{~J} \mathrm{~s}\right), c$ is the speed of light $(c=2.998$ $\left.\times 10^{8} \mathrm{~m} / \mathrm{s}\right)$, and $k$ is Boltzmann's constant $(k=1.3807$ $\times 10^{-23} \mathrm{~J} / \mathrm{K}$ ). For a temperature $T$ ranging from 350 to $1000^{\circ} \mathrm{C}$, the curves of the spectral photon intensity of an ideal source $\left(\varepsilon_{\lambda}=1\right)$, illustrated in Fig. 4 , reach a peak at wavelength values between 2.25 and $4.65 \mu \mathrm{m}$. Hence, 3- to $5-\mu \mathrm{m}$ IR cameras are the most suitable sensors to benefit from the maximum signal level. When compared to the NIR spectral band $(0.75$ to $1.1 \mu \mathrm{m})$, the intensity value for a temperature value of $350^{\circ} \mathrm{C}$ decreases by a ratio of $10^{6}$. Despite these low-illumination conditions, a temperature

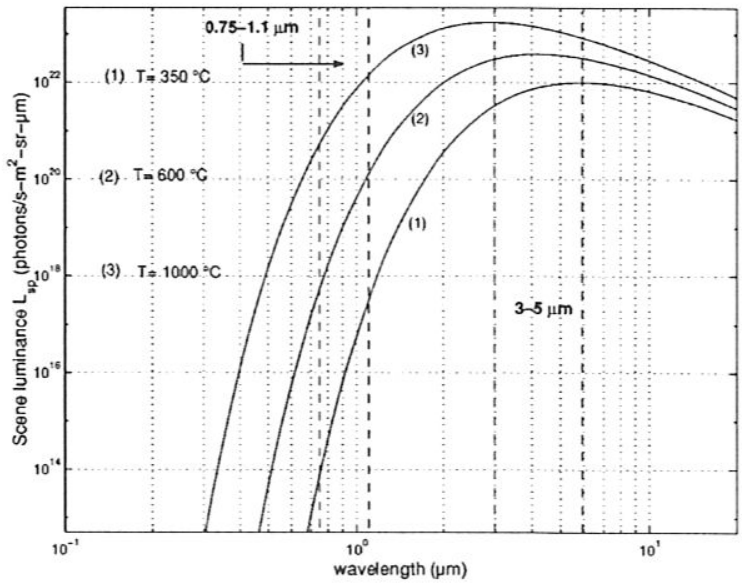

Fig. 4 Planck's spectral photon intensity. 
measurement can be performed. These intensity values also decrease with the object emissivity (see discussion in Sec. 4.2.1).

In the load displacement situation, the target appears as a pattern composed of eight reflective patches each $50 \mathrm{~mm}$ in diameter, enabling the estimation of the load orientation (see Fig. 2). The targets must be illuminated with a sufficient intensity $L_{e}$ from the IR source mounted inside the VSU to distinguish their reflected intensity $L_{\mathrm{sr}}$ from the environmental intensity. Moreover, the reflection coefficient $\rho$ must also be maximized, as shown in the following relation:

$L_{\mathrm{sr}}(\lambda)=\rho(\lambda) \tau_{e}(\lambda) L_{e}(\lambda)$,

where $\tau_{e}$ is the environment transmittance.

For example, a target $(\rho=0.46$ and a lozenge area $A_{\text {target }}=0.01 \mathrm{~m}^{2}$ ) placed at a maximal distance $d_{s}$ of $10 \mathrm{~m}$ and illuminated by a set of current technology LEDs $(0.88-\mu \mathrm{m}$ wavelength peak and an intensity of $900 \mathrm{~mW} / \mathrm{sr})$, leads to an irradiation $I_{0}$ equal to $3.52 \times 10^{-7} \mathrm{~W} / \mathrm{cm}^{2}$. This irradiation was measured with a radiometer in the NIR spectral band $(\Delta \lambda)$. The reflected intensity $L_{\mathrm{sr}}$ can be computed from the equation: $L_{\mathrm{sr}}(\lambda)=\left(I_{0} d_{s}^{2}\right) / A_{\text {target }}$. Moreover, from the relation $L_{\mathrm{sr}}(\lambda)=\int_{\Delta \lambda} L_{\mathrm{sp}}(\lambda, T)(h c) / \lambda \mathrm{d} \lambda$, a blackbody spectral radiant intensity $L_{\mathrm{sp}}$ that corresponds to a temperature of around $620^{\circ} \mathrm{C}$ (see Fig. 4) is computed. From Eq. (2) and using an environmental transmittance of 1 (see Sec. 2.2), the LED intensity $L_{e}$ corresponds to an equivalent blackbody temperature of about $680^{\circ} \mathrm{C}$. These temperature ranges correspond to the temperature of an overheating situation.

\subsection{Transmittance of the Intensity by the Environment $\left(\tau_{e}\right)$}

The physical environmental conditions introduced by fog in nonpressurized aircraft cargo compartments involve a spectral transmittance modification illustrated by the following Beer-Lambert relation:

$\tau_{e}\left(\lambda, d_{s}\right)=\exp \left[-\gamma_{e}(\lambda) d_{s}\right]$,

where $d_{s}$ is the distance between the scene intensity source and the VSU, while $\gamma_{e}$ denotes the extinction coefficient defined by the sum of the absorption $\alpha_{e}$ and the diffusion $\beta_{e}$ coefficients. The fog radius $r$ of particles is smaller ${ }^{13}$ than $10 \mu \mathrm{m}$, and the distance $d_{s}$ ranges between 1 and 15 $\mathrm{m}$. We suppose that fog is not absorbent $\left(\alpha_{e}=0\right)$ and the Mie theory provides the diffusion coefficient $\beta_{e}$ of an aerosol $^{14}$ for a mean wavelength value $\lambda$ of $0.88 \mu \mathrm{m}$. For these values, the fog transmittance coefficient $\tau_{e}$ decreases with the radius of particles and their distance, as illustrated in Fig. 5.

During the take-off phase, fog can be considered as a perturbation in load movements and temperature measurements. In the worst case (at a distance of $10 \mathrm{~m}$ and with a radius of particles greater than $10 \mu \mathrm{m}$ ), the transmittance coefficient is close to zero and the intensity is not transmitted. Preliminary tests in a cargo area show a quick transition phase between no fog $\left(\tau_{e}=1\right)$ and fog $\left(\tau_{e} \approx 0\right)$. There-

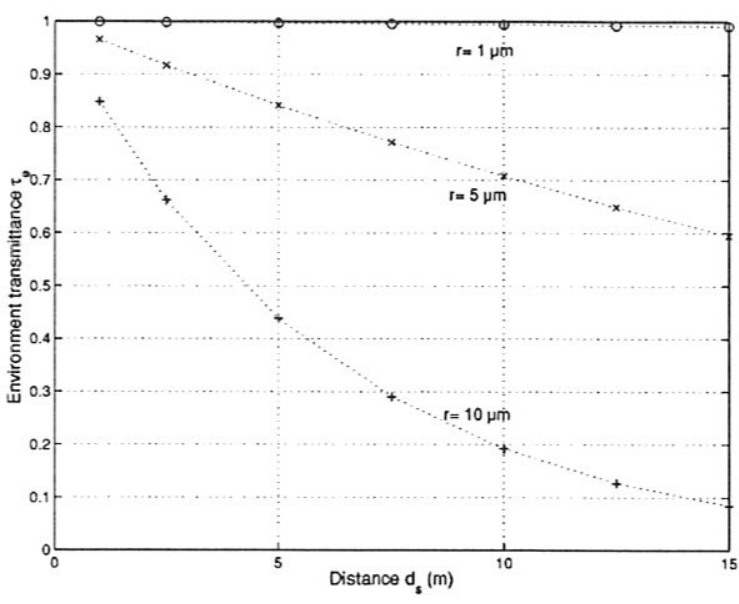

Fig. 5 Nonabsorbent fog transmittance.

fore, except during the take-off phase, we consider the environment transmittance as a constant value $\left(\tau_{e}=1\right)$.

\subsection{VSU Performance}

The purpose of this section is to verify whether the VSU is able to measure the scene intensity and to analyze the wavelength influence on the spatial resolution.

\subsubsection{VSU output signal}

Ideally, during the integration time $t_{i}$, a VSU associated with a frame video grabber (see Fig. 3 ) produces a digital signal $I_{D}$ given by

$I_{D}=K_{D} K_{c} t_{i} K_{o} \int_{\Delta \lambda} \tau_{e}(\lambda) \tau(\lambda) L_{s}(\lambda) \eta_{e}(\lambda) \mathrm{d} \lambda$,

where $\tau_{w}, \tau_{f}$, and $\tau_{o}$ are, respectively, the window glass, the NIR filter, and the optical system transmittances. The optical system concentrates photons on the CCD detector with a coefficient $K_{o}$. If the source is focused, $K_{o}$ is given by $\left(\pi A_{d}\right) /\left(4 N^{2}\right) \cos \left(\theta_{d}\right), N$ is the $F$-number, and $A_{d}$ is the effective sensitive area of the detector. This area is the pixel area $A_{\text {pix }}$ times the fill factor. Angle $\theta_{d}$ is the angle between the source camera axis and the normal direction of the detector. Microlenses can increase the effective area by an amplification $K_{m l}$ between 2 and 3. The detector quantum efficiency (ratio of electrons to incident photon flux), $\eta_{e}$, creates electrons (electronic charge $q$ ) at each collection site. Next, the charges are transferred simultaneously with a charge transfer efficiency $(C T E \approx 1)$ into an output register. Before the next parallel transfer, the register drives each of its charge packets to a floating diode acting as a capacitor $C_{c}$. The voltage difference $V_{c}$ of the capacitor is amplified with a gain $G_{c}$ and the final value is proportional to the number of electrons collected at each CCD pixel with a coefficient $K_{c}=\left(G_{c} q\right) / C_{c}$. Finally, the analog signal $V_{c}$ generated by the VSU (the full range value is termed $V_{c \mathrm{FR}}$ ) is converted into a digital signal $I_{D}$ with a resolution of $n$ bits and amplified with a gain $G_{D}$. This conversion intro- 
duces a coefficient $K_{D}=\left(G_{D} 2^{n}\right) / V_{c \text { FR }}$. For a dynamic range of $48 \mathrm{~dB}$, the number of bits $n$ is equal to 8 .

From Eq. (4), the coefficient $K_{o}$ must be chosen as large as possible (i.e., $N$ must be small and $A_{d}$ large) to maximize the output signal $I_{D}$. The problem of combining fire temperature and displacement measurements requires finding a trade-off between a large detector area $A_{d}$ to improve the output signal and a small one to respect a particular requirement for a spatial resolution of $50 \mathrm{~mm}$ at a distance of $15 \mathrm{~m}$ (see discussion in Sec. 2.3.2). An opposite approach can be applied to the $F$-number $N$. Given that the area of a classical CCD detector with a good resolution is around 8.6 $\times 8.6 \mu \mathrm{m}^{2}$, a low $F$-number $(N=1.4)$ is necessary to increase the number of photons.

Moreover, the quantum efficiency can also be improved by using a back-illuminated NIR optimized by antireflection coating. However, low-cost detectors are generally front-illuminated and their quantum efficiency in the NIR spectral band is often lower than $15 \%$. To compensate for the low-level scene spectral intensity (see Sec. 2.1) combined with low quantum efficiency in the NIR spectral band, a long integration time $t_{i}$ is required to improve the signal for temperature measurements. Conversely, to prevent the saturation of the CCD collection site in fire situations, short integration times must be used. Generally, the integration time $t_{i}$ can vary from $20 \mathrm{~ms}$ to $1 \mathrm{~s}$. Smaller values can be reached using shutter speeds equal to $1 / 125$, $1 / 250, \ldots, 1 / 10,000 \mathrm{~s}$. Before discussing the integration time selection, the camera resolution is studied to estimate the required detector area $A_{d}$ in the NIR spectral band. The camera resolution is described by the modulation transfer function (MTF), which is the camera amplitude response to sine wave inputs.

\subsubsection{VSU MTF}

The complete MTF of the VSU is defined by the product of the optic MTF $\left(\mathrm{MTF}_{o}\right)$ and the detector MTF $\left(\mathrm{MTF}_{d}\right)$, that is,

$\mathrm{MTF}=\mathrm{MTF}_{o} \times \mathrm{MTF}_{d}$.

The $\mathrm{MTF}_{o}$ in the horizontal or vertical direction of a radial symmetrical optical system with a clear circular diffractionlimited aperture illuminated monochromatically is given by ${ }^{15}$

$\operatorname{MTF}_{o}=\frac{2}{\pi}\left\{\cos ^{-1}\left(\frac{f_{s}}{f_{\mathrm{oc}}}\right)-\left(\frac{f_{s}}{f_{\mathrm{oc}}}\right)\left[1-\left(\frac{f_{s}}{f_{\mathrm{oc}}}\right)^{2}\right]^{1 / 2}\right\}$,

where $f_{\mathrm{oc}}$ is the cutoff frequency of the optical system $\left[f_{\circ c}=1 /(N \lambda)\right]$. In the NIR spectral band, as $N$ is equal to $1.4, \mathrm{MTF}_{o}$ is close to 1 , and it is the detector that limits the highest input spatial frequency. In this case, the VSU MTF can be approximated by

$\mathrm{MTF} \approx \mathrm{MTF}_{d}$.

The $\mathrm{MTF}_{d}$ depends on the geometry (pixel size) and on spectral considerations in the NIR spectral band. Owing to photon absorption occurring at increasing depths along the

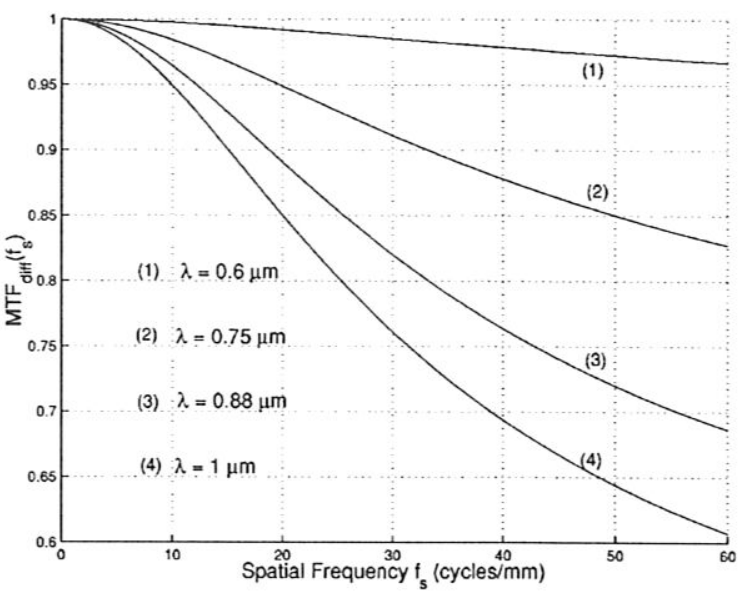

Fig. $6 \mathrm{MTF}_{\mathrm{ditf}}$ versus $f_{s}$ for different wavelengths.

substrate detector depth, an electron generated in the substrate will experience a 3-D random walk until it recombines or reaches the edge of neighboring depletion region. In the latter case, this phenomenon, called diffusion, creates a response that overlaps other pixels. Finally, the detector $\operatorname{MTF}\left(\mathrm{MTF}_{d}\right)$ can be expressed by the product of geometric $\operatorname{MTF}\left(\mathrm{MTF}_{\mathrm{geo}}\right)$ and the diffusion term $\left(\mathrm{MTF}_{\text {diff }}\right)$, as follows:

$\mathrm{MTF}_{d}=\mathrm{MTF}_{\mathrm{geo}} \times \mathrm{MTF}_{\mathrm{diff}}$.

The expression of $\mathrm{MTF}_{\mathrm{geo}}$ for a single nonrectangular CCD cell is given by ${ }^{12}$

$\operatorname{MTF}_{g e o}\left(f_{H_{s}}, f_{V_{s}}\right)=\left|\operatorname{sinc}\left(f_{H_{s}} \Delta x\right)\right|\left|\operatorname{sinc}\left(f_{V_{s}} \Delta y\right)\right|$,

where $f_{H_{s}}$ and $f_{V_{s}}$ denote, respectively, the horizontal and vertical frequencies of the sine input signal; and $\Delta x$ and $\Delta y$ represent the effective photosensitive detector sizes in the horizontal and vertical planes, and are equal to the pixel sizes times the fill factor. Note also that they can be modified by microlenses on the detector and an antialiasing filter.

The diffusion term MTF $_{\text {diff }}$ for a front-illuminated CCD detector can be written as ${ }^{16}$

$\operatorname{MTF}_{\mathrm{diff}}\left(\lambda, f_{s}\right)=\frac{1-\left\{\exp \left[-\alpha(\lambda) \chi_{p}\right]\right\} /\left[1+\alpha(\lambda) L_{o}\left(f_{s}\right)\right]}{1-\left\{\exp \left[-\alpha(\lambda) \chi_{p}\right]\right\} /\left[1+\alpha(\lambda) L_{n}\right]}$,

where $\alpha$ is the photon absorption coefficient that decreases with the increasing wavelength (see the formulas in the Appendix) and $\chi_{p}$ denotes the depletion width. Finally, the factor $L_{o}$ is the spatial frequency-dependent component of the diffusion length $L_{n}\left\{L_{o}=L_{n} /\left[1+\left(2 \pi L_{n} f_{s}\right)^{2}\right]^{1 / 2}\right\}$. Figure 6 shows the evolution of the $\mathrm{MTF}_{\text {diff }}$ term with different wavelengths and for a current CCD substrate with an epitaxial layer ( $\chi_{p}$ and $L_{n}$ are approximately 4 and $\left.7 \mu \mathrm{m}\right)$. For short wavelengths $(\lambda<0.6 \mu \mathrm{m}), \mathrm{MTF}_{\text {diff }}$ tends toward 1 


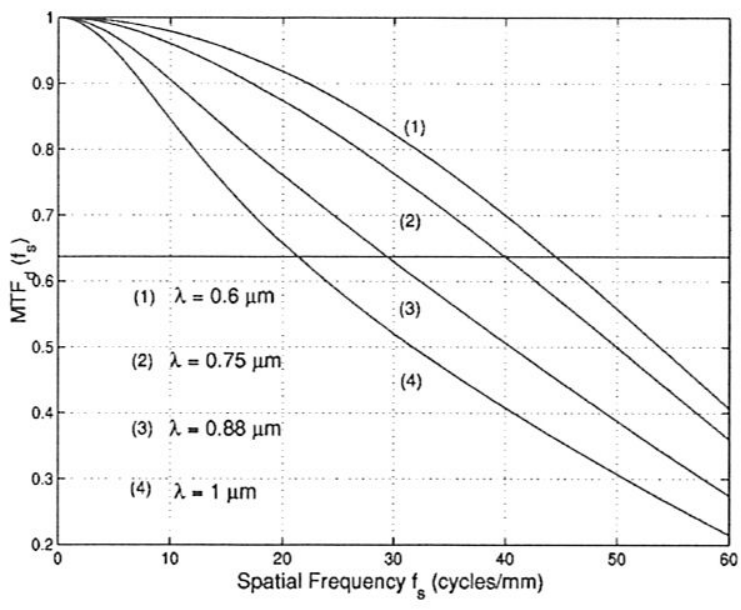

Fig. $7 \mathrm{MTF}_{d}$ versus $f_{s}$ for different wavelengths.

but decreases in the NIR spectral band $(\lambda>0.75 \mu \mathrm{m})$, and can no longer be neglected in the $\mathrm{MTF}_{d}$ relation. Figure 7 illustrates the evolution of the camera MTF with respect to the input frequency $f_{s}$ for several wavelengths and for an effective detector size of $8.6 \mu \mathrm{m}$.

For our metrology application, we set a criterion of a minimal contrast that corresponds to an MTF value of 0.637 , and for this value, we define the system band limit frequency $f_{c}$. In the visible spectral band, and in the horizontal direction, we note that this band-limit frequency is equal to $f_{c}=1 /(2 \Delta x)$. Applying this criterion in Fig. 7, the mean band-limit frequency is lower $\left(f_{c} \approx 30\right.$ cycles $\left./ \mathrm{mm}\right)$ in the NIR spectral band than in the visible region $\left(f_{c} \approx 45\right.$ cycles $/ \mathrm{mm}$ ). As a result, a factor of around 1.5 can be calculated on the band-limit frequency between the two spectral bands. We can consider that the sampler spatial ability of the system is different according to the spectral band. In conclusion, to preserve the same performance in terms of band-limit frequency in the NIR spectral band, we must apply a ratio of 1.5 to the detector size requirements.

As an illustration, a target spatial period $T_{\mathrm{ts}}$ of $50 \mathrm{~mm}$ at a maximal distance $d_{s}$ of $15 \mathrm{~m}$ was fixed. A focal length $f$ of $8 \mathrm{~mm}$ was chosen to achieve the required field of view. This period in object space corresponds to the spatial frequency $f_{d_{s}}$ in image space, which is equal to $f_{d_{s}}$ $=d_{s} /\left(f T_{t_{s}}\right)=37.5$ cycles $/ \mathrm{mm}$. To maintain our criterion, the spatial frequency $f_{d_{s}}$ must be greater than the system band-limit frequency $f_{c}$. In the visible region, the system band limit is equal to $f_{c}=1 /(2 \Delta x)$ and the detector size $\Delta x$ required is then equal to $13.3 \mu \mathrm{m}$. However, in the NIR spectral band, considering the $\mathrm{MTF}_{\text {diff }}$ term, the same value

Table 1 Parameter values of the measurement chain.

\begin{tabular}{cccccccccc}
\hline \hline$\tau_{e}$ & $\tau_{0}$ & $\tau_{w}$ & $f(\mathrm{~mm})$ & $N$ & $\Delta x(\mu \mathrm{m})$ & $\Delta y(\mu \mathrm{m})$ & $G_{c}$ & $G_{D}$ & $n$ \\
\hline 1 & 1 & 1 & 8 & 1.4 & 8.88 & 8.88 & 1 & 1 & 8 \\
\hline \hline
\end{tabular}

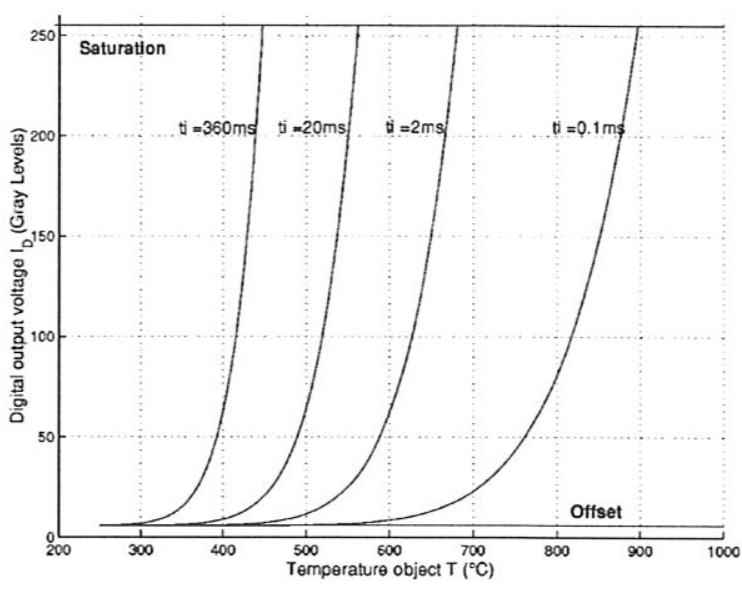

Fig. 8 Graph of $I_{D}$ versus $T$.

of system band-limit frequency can be obtained with detector sizes that are weighted by a factor of around 1.5 and the detector size value required must then be $8.88 \mu \mathrm{m}$.

\subsection{Conclusions}

From Eq. (4) and using the configuration of the measurement chain summarized in Table 1 , the value of $I_{D}$ can be computed as shown in Fig. 8.

In spite of a low spectral photon intensity $L_{s}$ at the temperature of $350^{\circ} \mathrm{C}$ (see Fig. 4) combined with a relatively low quantum efficiency of silicon $\eta_{e}$ in the NIR spectral band (see Fig. 9), a maximal integration time of $360 \mathrm{~ms}$ enables us to obtain an output signal $I_{D}$ greater than six times the output standard deviation $\left(\sigma_{I_{D}}\right)$ plus the output signal offset $\left(6 \times \sigma_{I_{D}}+\right.$ offset $)$. In the case of a digitizer of 8 bits, one gray level represents the standard deviation $\sigma_{I_{D}}$. This integration time value is compatible with a surveillance application. Conversely, a minimum time of $0.1 \mathrm{~ms}$ enables us to measure until reaching $900^{\circ} \mathrm{C}$. This tempera-

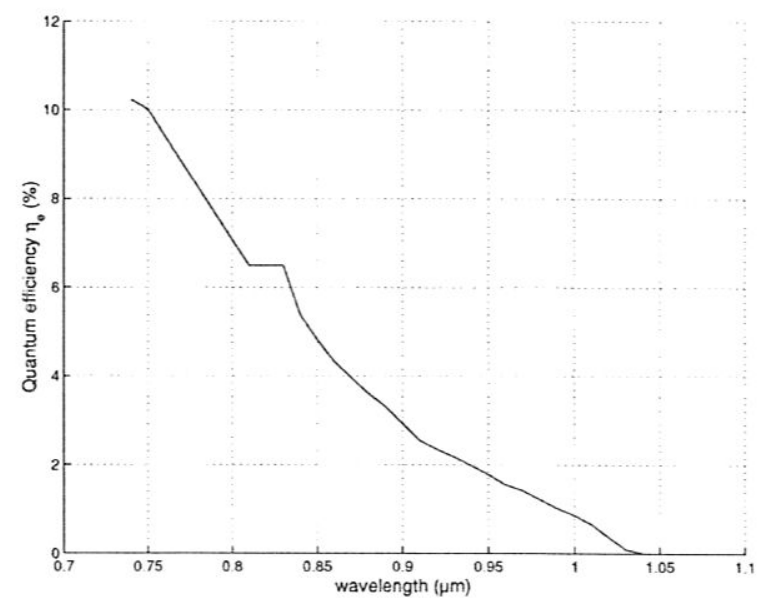

Fig. 9 Graph of $\eta_{e}$ versus $\lambda$. 
ture corresponds to an equivalent blackbody temperature of hydrocarbon fires such as benzene or kerosene. ${ }^{17}$ Moreover, in the case of illuminated targets assimilated to an equivalent blackbody temperature of around of $600^{\circ} \mathrm{C}$ and with the configuration of Table 1 , an integration time of $8 \mathrm{~ms}$ can be satisfactory to just saturate the image. At different distances, a possible subduing light in fog or smoke conditions can be detected and the target location can be performed rapidly. Finally, the problem of improving the output signal and satisfying the spatial resolution requirements can be accomplished using a classical size of CCD detector and by controlling the integration time. With the configuration of Table 1, the VSU would be able to measure (1) an equivalent blackbody temperature ranging from 350 to $900^{\circ} \mathrm{C}$ from overheating and flame without emitting light and with four integration times ranging from 360 to $0.1 \mathrm{~ms}$ and (2) transmissivity smoke and load displacements of 50 $\mathrm{mm}$ at a maximal observation distance of $15 \mathrm{~m}$ with emitting light on specific targets and an integration time of 8 $\mathrm{ms}$. To estimate the real integration time values, however, the sensor must be calibrated.

\section{VSU Models and Calibration Procedures}

In this section, the radiometric model and the associated calibration procedure are presented. The geometric model of the VSU sensor (optical lens, camera) and the frame grabber required for load localization is also determined and identified with a specific calibration procedure. Moreover, to check whether the loss of spatial resolution in the NIR spectral band is effective, some resolution measurements are performed.

According to the configuration of Table 1, we tested several kinds of low-cost CCD cameras that could be integrated in the VSU. They are based on a detector array of an $8.6(H)-\times 8.3(V)-\mu \mathrm{m}^{2}$ pixel surface. These cameras have a linear response in irradiation and are equipped with an NIR blocking filter. All experimental tests were carried out in a dark environment whose temperature was always close to $25^{\circ} \mathrm{C}$. Note that we assume that the detector temperature is constant. The influence of the temperature variations in a cargo compartment from -55 to $85^{\circ} \mathrm{C}$ on the radiometric and geometric models are being studied. Preliminary results for the geometric camera model can be found in Ref. 18 .

\subsection{Radiometric Model}

The radiometric model is the effective relation between the temperature $T$ of an ideal blackbody source and the digital output $I_{D}$ of the VSU. This model is usually determined with IR cameras, but it can be directly used with CCD cameras. In this section, the site-to-site variation of the pixel value that is due to sensor noise is neglected. It is considered in Sec. 4.3. From Eq. (4) and considering a very small wavelength range $(\Delta \lambda=0.35 \mu \mathrm{m})$, the integral can be computed to around an effective wavelength $\lambda_{e}$. And thus, the quantum efficiency $\eta_{e}$ and the atmosphere, window, filter, and optical system transmittances can be considered as constant. Only integration time $t_{i}$ and analog-todigital converter gain $G_{D}$ can be modified on-line and we obtain:

$I_{D}=A_{1} G_{D} t_{i} L_{s}\left(\lambda_{e}, T\right)$,

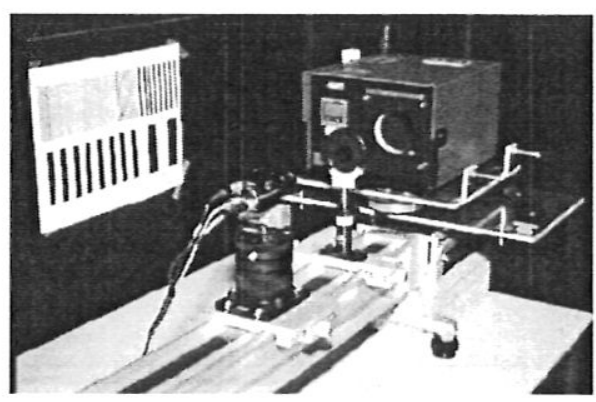

Fig. 10 Experiment for radiometric calibration.

where $A_{1}=\left(\tau_{e} \tau_{w} \tau_{f} \tau_{o} K_{o} K_{c} \eta_{e} 2^{n}\right) / V_{c \mathrm{FR}}$.

In a second step, using Planck's blackbody radiation law [Eq. (1)] with an emissivity $\varepsilon_{\mathrm{BB}} \approx 1$ and introducing a variable $C$ to model the camera continuous output voltage, we get

$I_{D}=\frac{A_{2} G_{D} t_{i}}{\exp (B / T)-1}+C$,

where $A_{2}$ and $B$ are constants given, respectively, by $A_{2}$ $=A_{1} \varepsilon_{\mathrm{BB}} C_{3} \lambda_{e}^{-4}$ and $B=C_{2} / \lambda_{e}$. Finally, Eq. (12) describes the radiometric model with three parameters $\left(A_{2}, B\right.$, and $\left.C\right)$ that are determined during the calibration procedure described in the next section.

\subsection{Radiometric Calibration Procedure}

The camera is directly illuminated by a blackbody (see Fig. 10) at a temperature ranging from 300 to $550^{\circ} \mathrm{C}$. A blackbody cavity with a high aperture $(65 \mathrm{~mm})$ is used to calibrate a maximum of pixels $(280 \times 380$ pixels) (see the uniform region in Fig. 11). Using the relation $A_{t s}=d_{s} A_{d s} / f$ $\left(f=8 \mathrm{~mm}\right.$ and $\left.d_{s}=15 \mathrm{~m}\right)$, the calibrated detector area $A_{d s}$ of $280 \Delta x \times 380 \Delta y \mathrm{~mm}$ corresponds to a target area $A_{t s}$ of $4.3 \times 6.1 \mathrm{~m}$. Above $550^{\circ} \mathrm{C}$, a blackbody with a smaller aperture is used.

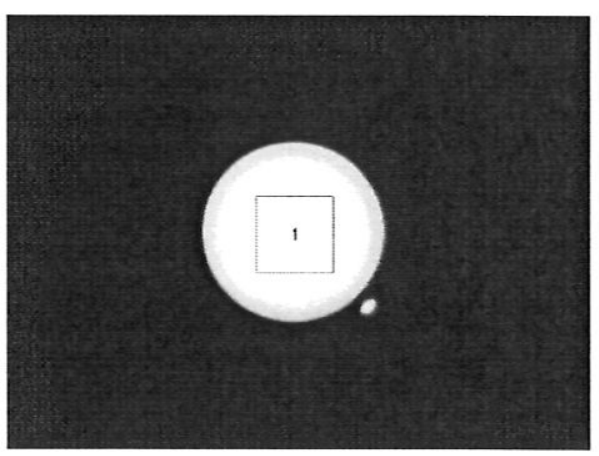

Fig. 11 Image of blackbody source over $350^{\circ} \mathrm{C}$ in the NIR spectral band. The square (1) represents the uniform blackbody region where the calibration is effective. 


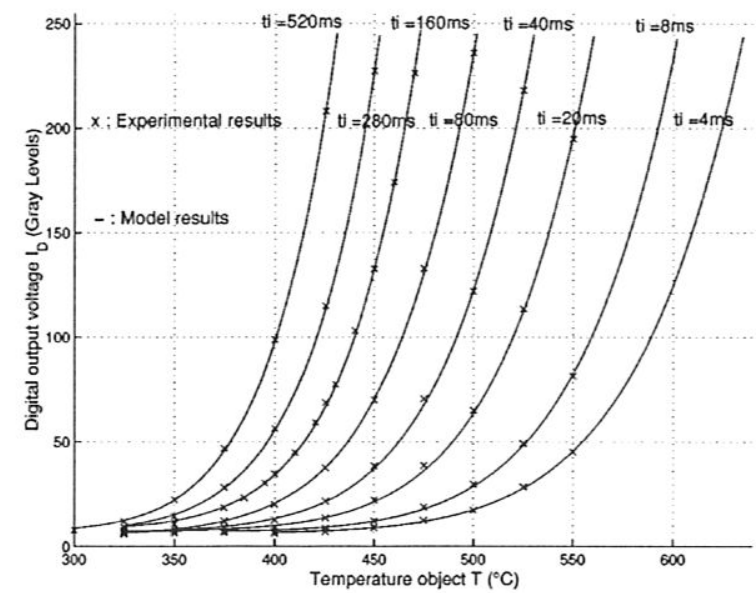

Fig. 12 Temperature calibration for $t_{i} \in[4 \mathrm{~ms}, 520 \mathrm{~ms}]$.

\subsubsection{Calibration results}

Figures 12 and 13 show experimental and model points ( $T$ and $I_{D}$ ) of CCD camera for optical values given in Table 1 and integration times $t_{i}$ ranging from 520 to $0.1 \mathrm{~ms}$. Here $T$ is the temperature indicated by the blackbody temperature controller, and $I_{D}$ is the digital mean value, which is computed in the blackbody uniform region for several images (see Fig. 11). The parameters $A_{2}, B$, and $C$ of Eq. (12) are estimated from the experimental values and provided in Table 2.

As an illustration of the obtained performances, Fig. 14 shows the VSU sensitivity $S_{D}$ [derivative of the model of Eq. (12)]. Instead of using the classical definition of the noise equivalent temperature differences (NETDs) for an SNR of 1 , a generalized NETD is computed using the relation $\mathrm{NETD}_{G}=\left(\sigma_{I_{D}}\right) /\left(\mathrm{d} I_{D} / \mathrm{d} T\right)_{T=T_{0}}$ for $T_{0}$ ranging from detection to saturation temperatures. Figure 15 shows the NETD $_{G}$ and we note that an integration time of $360 \mathrm{~ms}$ enables us to measure a temperature ranging from 350 to

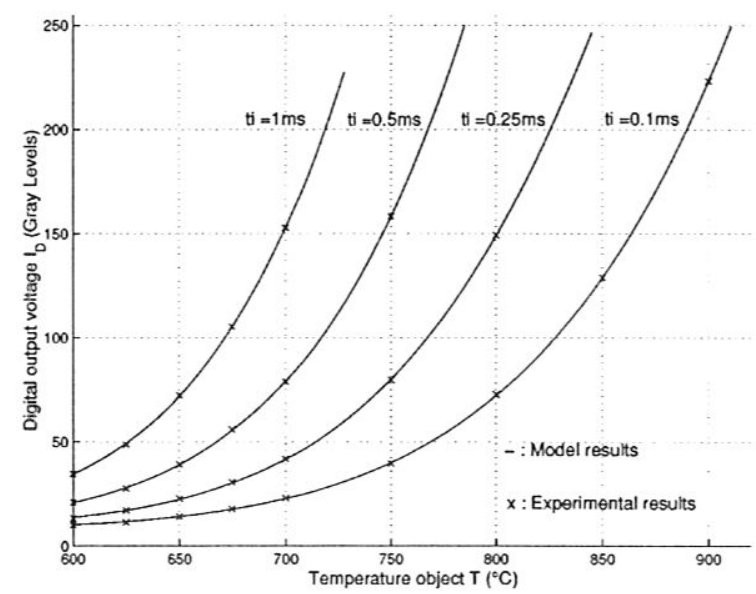

Fig. 13 Temperature calibration for $t_{i} \in[0.1 \mathrm{~ms}, 1 \mathrm{~ms}]$.
Table 2 Parameters values of the model of Eq. (12).

\begin{tabular}{lcc}
\hline \hline$A_{2}$ & $B$ & $C$ \\
\hline $1.025 \times 10^{+12}$ & $1.517 \times 10^{+4}$ & 7.94 \\
\hline \hline
\end{tabular}

$445^{\circ} \mathrm{C}$ with a generalized NETD decreasing from 2.4 to $0.1^{\circ} \mathrm{C}$. In the same temperature range, we also notice that the sensitivity rises rapidly $\left(0.3\right.$ to 7.25 gray levels $\left./{ }^{\circ} \mathrm{C}\right)$ with an increase of $95^{\circ} \mathrm{C}$. This high sensitivity value involves a minimum of four integration times between 0.1 and $360 \mathrm{~ms}$ (i.e., four images) to cover the considered temperature range. This high number of integration times can be explained by the small well capacity due to low detector size. We now address the problem of finding the lowest integration time required to reach the specifications.

\subsubsection{Comparison of camera performance}

Applying the calibration procedure to other cameras based on the same detector (both user gain and automatic gain are turned off), the minimal integration time $t_{i}$ is established to detect a temperature $T_{m}$ of $350^{\circ} \mathrm{C}\left(I_{D}>6 \sigma_{I_{D}}+\right.$ offset $)$ and to measure it with a minimal instantaneous uncertainty $\sigma_{T}$ equal to $\pm 3 \operatorname{NETD}_{G}$ (see Figs. 16 and 17). Moreover, in a dark environment, the amplitude of the output signal fluctuations of each camera is measured, and finally the camera dynamic ranges (DRs) are computed and provided in Table 3. From Fig. 16, the integration times enabling detection are between 100 and $240 \mathrm{~ms}$ depending on the cameras. However, Fig. 17 shows that the decrease of the instantaneous uncertainty is extremely small and an optimal integration time can be computed (for camera 1, this integration time is of around $360 \mathrm{~ms}$ ). The minimal integration times enabling detection and measurement are summarized in Table 3. By introducing an output gain $G_{D}$ greater than 1 , these values can be improved, but the output signal standard deviation is also increased. For cameras based on detectors with a smaller area than in the simulation, the performance is similar because microlenses improve photon

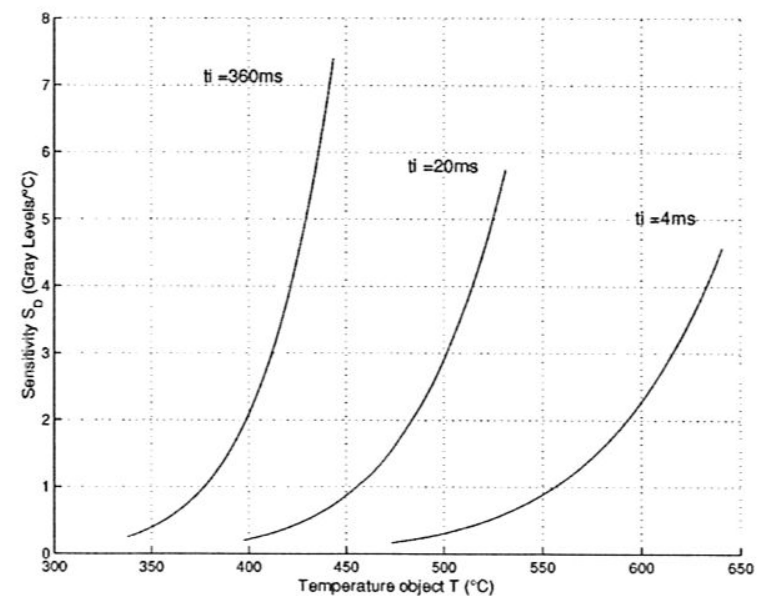

Fig. 14 Evolution of $S_{D}$ versus $T$. 


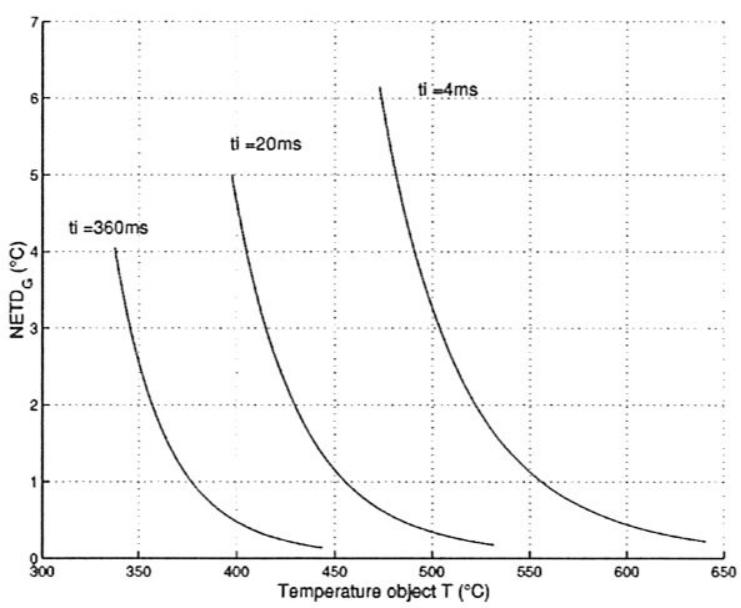

Fig. 15 Generalized NETD $_{G}$ versus $T$.

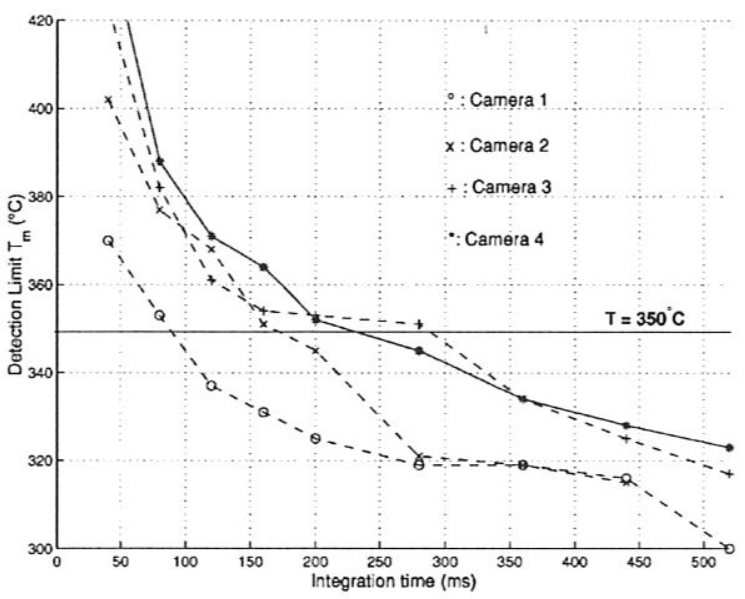

Fig. 16 Evolution of $T_{m}$ versus $t_{i}$.

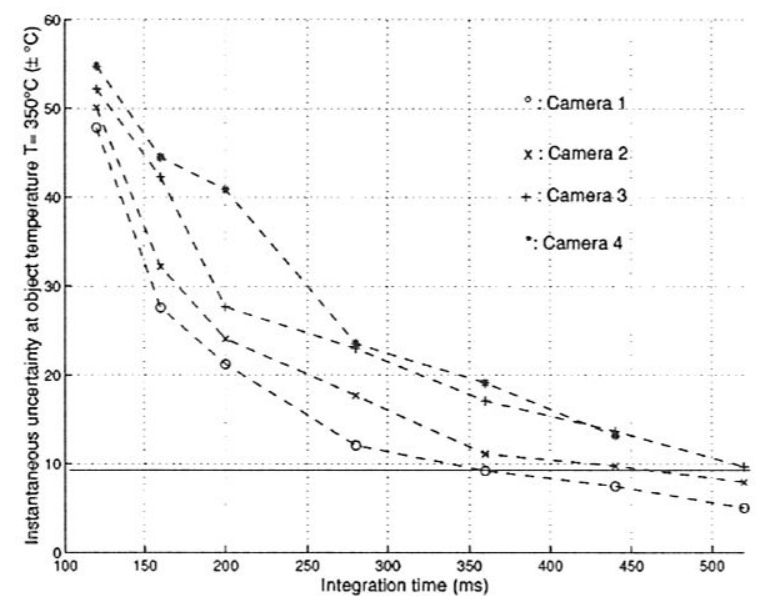

Fig. 17 Evolution of $\sigma_{T}$ versus $t_{i}$.
Table 3 Dynamic and integration time ranges.

\begin{tabular}{ccccc}
\hline \hline & Camera 1 & Camera 2 & Camera 3 & Camera 4 \\
\hline DR (dB) & 48 & 50 & 48 & 47 \\
$t_{i}(\mathrm{~ms})$ & 100 to 360 & 160 to 360 & 240 to 400 & 240 to 400 \\
\hline \hline
\end{tabular}

collection. The progressive scan array architecture is used in camera 4 , which supplies an advantage in motion measurement and improves the vertical resolution. The integration time mode, termed the field in cameras 1,2 , and 3 , which enables us to sum two frames, also enhanced the measurement efficiency in comparison to that of camera 4 . The performance differences between these three cameras can be explained by a different internal electronics (amplifiers and filters).

\subsection{Geometric Model}

An important task in 3-D computer vision is camera calibration, especially when metric information is required for applications involving accurate dimensional measurements. Camera calibration consists of determining the elements that govern the relationship between the 2-D image that a camera perceives and the 3-D information of the imaged object (see Fig. 18). Generally this is performed by choosing a parametric model for the camera and by estimating the parameters of this model by means of a calibration procedure.

This section describes the geometric model of the VSU sensor, which is based on the "pinhole" model described in Ref. 19. For our application involving accurate tridimensional (3-D) measurements, a model taking into account the optical distortions is required.

\subsubsection{Distortion-free model}

Let $\widetilde{\mathbf{M}}=(x y z 1)^{T}$ be the homogeneous coordinates of a 3-D point in the world reference frame (fixed arbitrarily), and let $\widetilde{\mathbf{m}}=(u v 1)^{T}$ be the pixel homogeneous coordinates of its image projection (see Fig. 19). Using the classical distortion-free pinhole model, the relation between $\widetilde{\mathbf{M}}$ and $\widetilde{\mathbf{m}}$ is given by

$\widetilde{\mathbf{m}} \cong \mathbf{P} \widetilde{\mathbf{M}}=\mathbf{K}\left(\begin{array}{ll}\mathbf{R} & \mathbf{t} \\ \mathbf{0}^{T} & 1\end{array}\right) \tilde{\mathbf{M}}$,

where $\cong$ is the projective equality, i.e., the equality defined up to a nonnull scale factor; $\mathbf{P}$ is the perspective projection matrix; $\mathbf{K}$ is the intrinsic matrix, which depends on four intrinsic parameters $\left(u_{0}, v_{0}, \alpha_{u}, \alpha_{v}\right) ; u_{0}$ and $v_{0}$ are the coordinates of the principal point, given by the intersection of the optical axis with the retinal plane; $\alpha_{u}=f k_{u}$ and $\alpha_{v}$ $=f k_{v}$ ( $f$ is the focal length in units of length, and $k_{u}$ and $k_{v}$

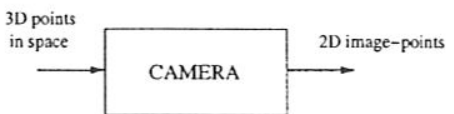

Fig. 18 Camera performs a 3-D-to-2-D transformation 


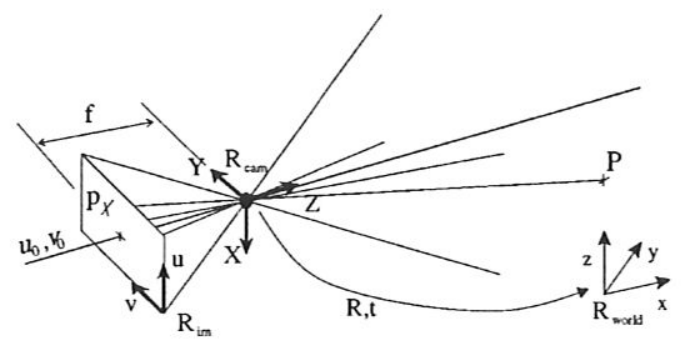

Fig. 19 Pinhole camera model.

are the number of pixels per unit of length along the $u$ and $v$ axes); $\mathbf{R}$ and $\mathbf{t}$ are extrinsic parameters and are the rotation and translation matrices that relate the world reference frame to the camera reference frame (see Fig. 19). Equation (13) relating the 3 -D point $(x y z)^{T}$ to its image projection $(u v)^{T}$ can be written as

$u=u_{0}+\alpha_{u} \frac{r_{11} x+r_{12} y+r_{13} z+t_{x}}{r_{31} x+r_{32} y+r_{33} z+t_{z}}$,

$v=v_{0}+\alpha_{v} \frac{r_{21} x+r_{22} y+r_{23} z+t_{y}}{r_{31} x+r_{32} y+r_{33} z+t_{z}}$,

where $\mathbf{R}=\left\{r_{i j}\right\}$ and $\mathbf{t}=\left(t_{x} t_{y} t_{z}\right)^{T}$.

Under the assumption that pixels are adjacent in the CCD array, $\alpha_{u}$ and $\alpha_{v}$ are

$\alpha_{u}=\frac{f}{\Delta x(\lambda)}, \quad \alpha_{v}=\frac{f}{\Delta y(\lambda)}$,

where $f$ is the focal length, and $\Delta x$ and $\Delta y$ are the effective size of the detector. The size dependence on wavelength $(\lambda)$ is discussed in Sec. 2.3.2.

\subsubsection{Model with distortion}

Several authors ${ }^{20,21}$ have shown that distortion-free models are not accurate enough for applications involving accurate dimensional measurements.

Calling $\mathbf{k}=\left(u_{0} v_{0} \alpha_{u} \alpha_{v}\right)^{T}$ the intrinsic parameter vector and $\mathbf{d}=\left(r_{1} r_{2} r_{3}\right)^{T}$ the distortion parameter vector (we consider only the first three terms of radial distortion), the relation between a 3-D point $\mathbf{M}=(x y z)^{T}$ in the world reference frame and the pixel coordinates $\mathbf{m}=\left(u_{d} v_{d}\right)^{T}$ of its image projection can be written as

$\mathbf{m}=g(\mathbf{k}, \mathbf{d}, \mathbf{R}, \mathbf{t}, \mathbf{M})$,

where $g()$ is a nonlinear function whose expression can be found in Ref. 22.

Finally, the geometric model with distortion is nonlinear and involves six extrinsic parameters (three independent parameters for rotation $\mathbf{R}$ and three for translation $\mathbf{t}$ ) and seven intrinsic parameters $\left(u_{0}, v_{0}, \alpha_{u}, \alpha_{v}, r_{1}, r_{2}, r_{3}\right)$. Those unknown parameters will be computed using a calibration procedure described in Sec. 3.5.

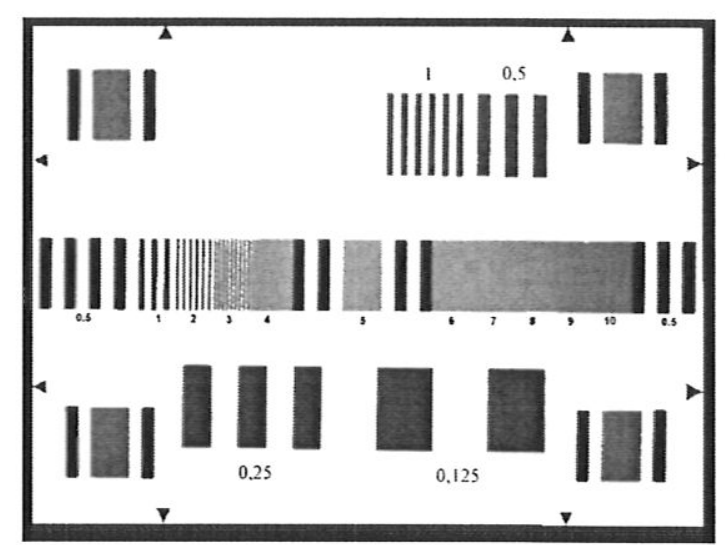

Fig. 20 Bar test target.

\subsection{MTF Measurement}

To analyze the wavelength dependence of the camera resolution, we compared the band-limit frequency $f_{c}$ in visible and in the NIR spectral bands. Although the camera MTF is defined for sine wave inputs, the square-wave test target (see Fig. 20) is the most popular for IR systems and the contrast transfer function (CTF) is established:

$\operatorname{CTF}\left[f_{s}(k)\right]=\frac{V_{c \max }\left[f_{s}(k)\right]-V_{c \min }\left(f_{s}\right)(k)}{V_{c \text { max }}\left[f_{s}(k)\right]+V_{c \min }\left[f_{s}(k)\right]}$

where $V_{c \max }$ and $V_{c \min }$ are, respectively, the maximum and minimum analog outputs of the VSU for the white and black bar test (see Fig. 21); and $f_{s}(k)$ are the spatial frequencies of the square-wave target in image space. The fundamental spatial frequency $f_{s}(0)$ is 4.5707 cycles $/ \mathrm{mm}$ and the other frequencies $f_{s}(k)$ are equal to $2 k f_{s}(0)$, where $k$ is an integer ranging from 1 to 10 . Hence, the highest frequency is given by $20 \times f_{s}(0)=90 \mathrm{cycles} / \mathrm{mm}$. In our experiment, we have hidden the bar with frequencies higher than $10 \times f_{s}(0)=45.707 \mathrm{cycles} / \mathrm{mm}$. Then, they are not aliased down to lower frequencies. Under this assumption, the sine wave amplitude at frequency $f_{s}(k)$ is expressed as an infinite sum of the square-wave amplitude ${ }^{15}$ and the relation between the measured CTF and the estimated MTF is given by

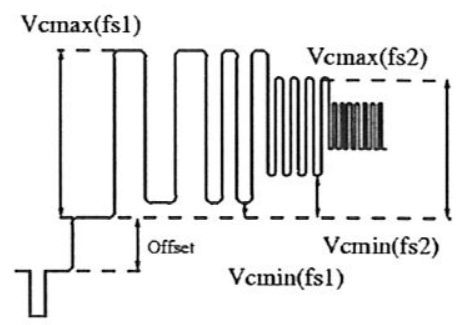

Fig. 21 Definition of the maximal $V_{c \max }$ and minimal $V_{c \text { min }}$ analog output VSU, respectively. 


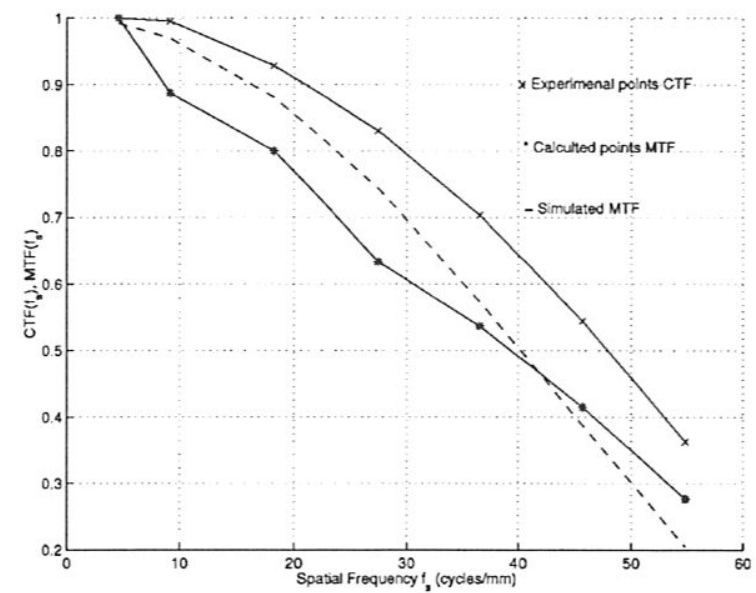

Fig. 22 Measured CTF, calculated MTF, and simulated MTF in the visible region.

$$
\begin{aligned}
\operatorname{MTF}\left[f_{s}(k)\right]= & \frac{\pi}{4} \mid \operatorname{CTF}\left[f_{s}(k)\right]+\frac{\operatorname{CTF}\left[f_{s}(3 k)\right]}{3} \\
& -\frac{\operatorname{CTF}\left[f_{s}(5 k)\right]}{5}+\frac{\operatorname{CTF}\left[f_{s}(7 k)\right]}{7} \\
& +\frac{\operatorname{CTF}\left[f_{s}(11 k)\right]}{11}+\text { irregular terms } \mid
\end{aligned}
$$

We note that for spatial frequencies $f_{s}(k)$ above $1 / 3$ of frequency computed to $\mathrm{MTF}=0$, Eq. (17) can be simplified and it is equal to $(\pi / 4) \operatorname{CTF}\left[f_{s}(k)\right]$.

As shown in Figs. 22 and 23, the CTF response, measured with Eq. (16), is lower in the NIR spectral band than in the visible region. From Eq. (17) many square-wave responses must be measured to estimate the MTF. In fact, this condition is fulfilled only for the fundamental frequency, and for other frequencies, the computations are approxi-

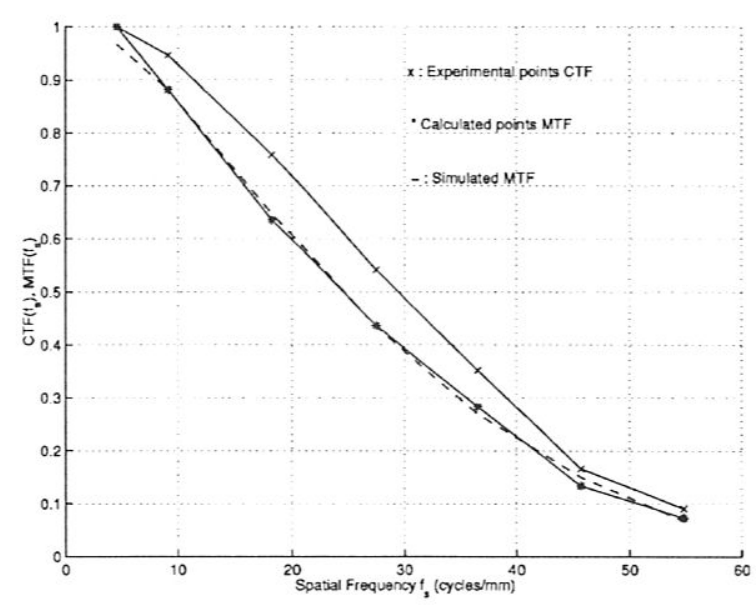

Fig. 23 Measured CTF, calculated MTF, and simulated MTF in the NIR spectral band.

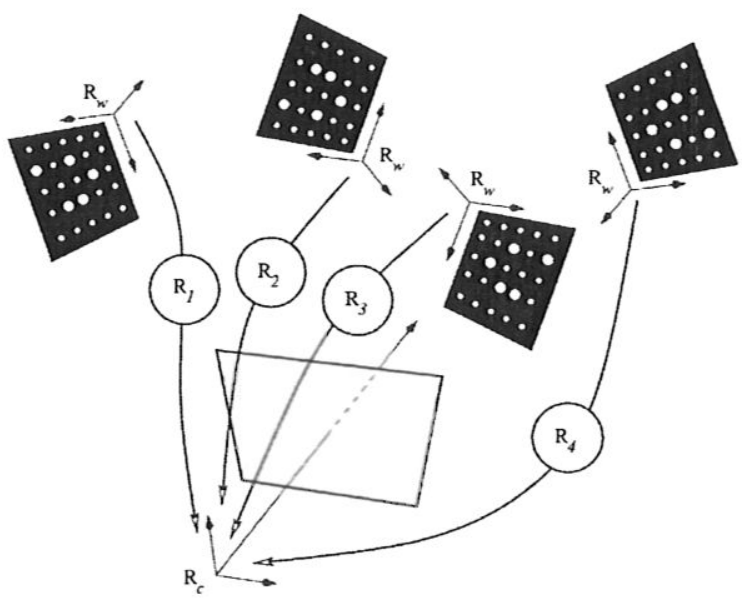

Fig. 24 Calibration of a camera from multiple views of a planar object.

mated. However, the loss of MTF obtained in the NIR rather than the MTF produced in visible is confirmed. Finally, the VSU simulated MTF, expressed in visible region by Eq. (9), is also plotted in Fig. 22 with an estimated detector width of $14.1 \mu \mathrm{m}$. Likewise, Eq. (8) $\left(L_{n}\right.$ $=8.701 \mu \mathrm{m}$ and $\chi_{p}=4.01 \mu \mathrm{m}$ in the $\mathrm{MTF}_{\mathrm{diff}}$ term) gives the VSU-simulated MTF in the NIR spectral band, which is fitted in Fig. 23. Hence, the estimated band-limit frequency $f_{c}$ (for an MTF value of 0.637 ) is of around 30 cycles $/ \mathrm{mm}$ in the visible region and in the NIR spectral region is only 20 cycles $/ \mathrm{mm}$. The factor of 1.5 between the band-limit frequencies in two spectral bands is confirmed.

From this result, a consequence can be derived in the geometric model, and particularly to Eq. (14), through the computation of an apparent spatial detector width $[\Delta x$ $\left.=1 /\left(2 f_{c}\right)\right]$. In the NIR spectral band, considering the MTF loss, it is equivalent to working with effective detector sizes 1.5 times greater. As a consequence, we must calibrate the geometric model in the NIR spectral band. Note that the apparent spatial size does not influence the capability of photon collection in Eq. (4).

\subsection{Geometric Calibration Procedure}

Calibrating a camera involves determining its intrinsic parameters and its position and orientation with respect to a world reference frame (see Sec. 3.3). The position of this world reference frame is chosen during the calibration procedure. For our application, this reference frame need not to be known in the cargo area because only relative measurements (load displacements) are estimated. Considering the MTF results (see Sec. 3.4), the VSU must be calibrated in the NIR spectral band.

We developed a flexible technique to easily calibrate a camera. ${ }^{22}$ This technique requires the camera to observe only a quasiplanar pattern shown at a few different orientations (see Fig. 24). The motion of the pattern need not be known and the pattern itself can be imprecise. We use a photogrammetric bundle adjustment approach to estimate together the intrinsic and extrinsic parameters and the 3-D points of the pattern. Now, consider $n$ images of a planar 
Table 4 Intrinsic and distortion parameters.

\begin{tabular}{ccccccc}
\hline \hline$u_{0}$ & $v_{0}$ & $\alpha_{u}$ & $\alpha_{v}$ & $r_{1}$ & $r_{2}$ & $r_{3}$ \\
\hline 237.7 & 351.2 & 964.4 & -969.9 & -0.375 & $6.78 \times 10^{-2}$ & 0.196 \\
\hline \hline
\end{tabular}

pattern made of $p$ points viewed by a camera (see Fig. 24). Let $\mathbf{m}_{i}^{j}$ be the coordinates of the projection of the $j$ 'th point $(j=1 \ldots p)$ of the $i$ 'th view $(i=1 \ldots n)$ onto the camera. From Eq. (15), we can deduce the following relation:

$\mathbf{m}_{i}^{j}=g\left(\mathbf{k}, \mathbf{d}, \mathbf{R}_{i}, \mathbf{t}_{i}, \mathbf{M}_{j}\right)$

There are four unknown intrinsic parameters, three unknown distortion parameters, $6 n$ unknown extrinsic parameters (three for each rotation $\mathbf{R}_{i}$ and three for each translation $\mathbf{t}_{i}$ ), and $3 p$ unknown 3-D coordinates $\mathbf{M}_{j}$ : that is a total of $7+6 n+3 p$ unknowns. Moreover, each 3-D point projection gives two relations [see Eq. (18)]. Thus, we have $2 n p$ equations and $7+6 n+3 p$ unknowns. Therefore, if $n$ and $p$ are large enough, there are more equations than unknowns (for instance, if $n=6$ and $p=48$, there are 576 equations for 187 unknowns), and we can estimate them by minimizing the sum of the 2-D algebraic distances between the projection of the $j^{\prime}$ 'th point of the $i$ 'th view onto the camera and the corresponding image point $\hat{\mathbf{m}}_{i}^{j}$ extracted in the $i$ 'th image:

$\min _{\theta} \sum_{i=1}^{n} \sum_{j=1}^{p}\left\|\hat{\mathbf{m}}_{i}^{j}-g\left(\mathbf{k}, \mathbf{d}, \mathbf{R}_{i}, \mathbf{t}_{i}, \mathbf{M}_{j}\right)\right\|^{2}$,

with $\theta=\left(\mathbf{k}, \mathbf{d}, \mathbf{R}_{1 \ldots n}, \mathbf{t}_{1 \ldots n}, \mathbf{M}_{1 \ldots p}\right)$. Minimizing Eq. (19) is a nonlinear minimization problem, which is solved with the Levenberg-Marquardt algorithm.

A more detailed description of the calibration procedure and a detailed discussion on accuracy evaluation can be found in Ref. 22. The VSU intrinsic parameters obtained by minimizing Eq. (19) are provided in Table 4.

\subsection{Conclusion}

In the calibration conditions, a temperature ranging from 350 to $900^{\circ} \mathrm{C}$ with a resolution of 25 to $0.4^{\circ} \mathrm{C}$ can be measured with integration times ranging from 360 to $0.1 \mathrm{~ms}$. The displacement measurement in the NIR spectral band involves a loss of spatial resolution of 1.5. A maximal resolution of $50 \mathrm{~mm}$ can be achieved for an observation distance $d_{s}$ of $12.2 \mathrm{~m}$ with a focal length of $8 \mathrm{~mm}$.

\section{Temperature Measurements}

This section contains a few examples of temperature measurements for some overheating or fire events. Accurate radiation thermometry measurements require a good understanding of the phenomenon of emissivity, the location of the source, and the surrounding environment. In aircraft cargo compartments, these characteristics are unknown and very difficult to estimate. Thus, their influence is analyzed by computing the resulting temperature uncertainty.

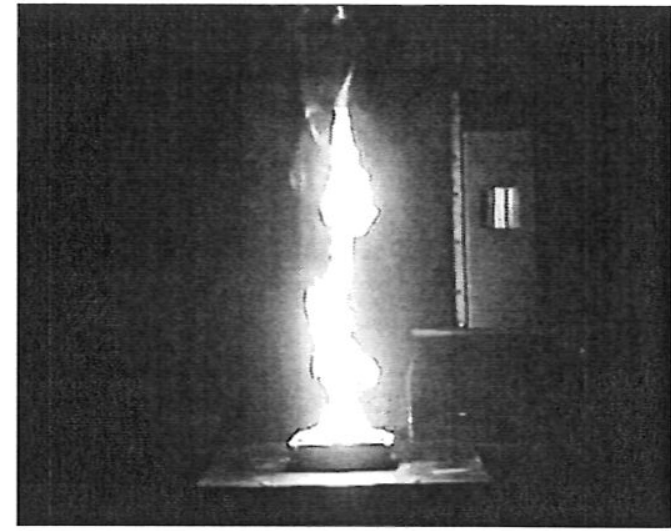

Fig. 25 Fire in visible light

\subsection{Examples and Temperature Relation}

In the laboratory, fire situations (see Fig. 25) are digitized with four integration times ranging from 360 to $0.1 \mathrm{~ms}$ and the result is illustrated in Fig. 26. From the digital value $I_{D}$ in the first nonsaturated image, an equivalent blackbody temperature of the phenomena is estimated using the relation

$T_{\mathrm{eq}}=\frac{B}{\ln \left[\left(A_{2} G_{D} t_{i} / I_{D}-C\right)+1\right]}$.

Above $600^{\circ} \mathrm{C}$, a flame situation is considered, and only an equivalent blackbody temperature without emissivity correction is given. Because the flame emissivity is wavelength dependent, the only realistic approximation is a unitary emissivity. In addition, the radiation emitted by the flame and by illuminated neighboring objects cannot be distinguished. All those parasite reflections may induce errors larger than several hundred degrees.

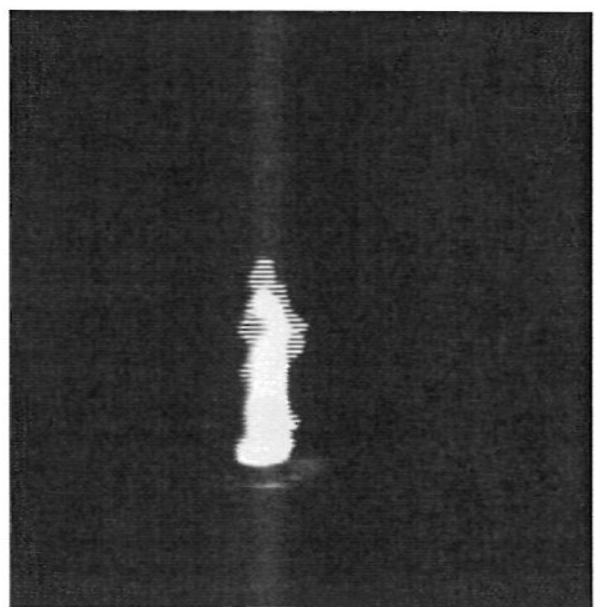

Fig. 26 Fire in NIR for $t_{i}=0.1 \mathrm{~ms}$ 
Table 5 Literature emissivity values.

\begin{tabular}{ccccc}
\hline \hline & Black Paint & Wood-Paper & Aluminum Paint & White Paint \\
\hline$\varepsilon$ & 0.97 to 0.89 & 0.9 to 0.8 & 0.7 to 0.5 & 0.4 \\
\hline \hline
\end{tabular}

Below $600^{\circ} \mathrm{C}$, in smoldering fire situations, the equivalent blackbody temperature can be corrected by the emissivity $(\varepsilon)$, the location of the source $\left[\cos \left(\theta_{d}\right)\right]$ and the environment transmittance $\left(\tau_{e}\right)$. To this end, Eq. (20) should be then modified as follows:

$T=\frac{B}{\ln \left[\left(A_{2} \varepsilon \tau_{e} \cos \left(\theta_{d}\right) G_{D} t_{i} / I_{D}-C\right)+1\right]}$.

Moreover, if the source of the scene is not under focus, the source-camera distance $\left(d_{s}\right)$ must be introduced in Eq. (21). Except for $\tau_{e}$, which can be estimated by the measurement of the reflection coefficient of a known remote target illuminated by an emitting IR source, it is difficult to control the other parameters. Therefore, their influence is studied.

\subsection{Influence of Critical Parameters}

\subsubsection{Emissivity}

In aircraft cargo compartments, the usual objects are often made of metal, wood, cardboard, plastic, and textiles. In the NIR spectral band, they are considered as opaque and gray bodies with a constant emissivity ranging from 0.4 to 0.97 (see Table 5) (Refs. 14 and 23). We made a copper sheet with four different emissivity areas (see Fig. 27) to check these literature values. The sheet is warmed up a temperature $T_{\text {ref }}$ of $425^{\circ} \mathrm{C}$, which is regulated by a thermocouple associated with a controller. This temperature enables us to obtain a maximal $I_{D}$ signal and it is much higher than room temperature $\left(25^{\circ} \mathrm{C}\right)$. Therefore, the latter can be neglected in radiometric Eq. (4). We also assume that no hot object is reflected on the sheet during the measurements.

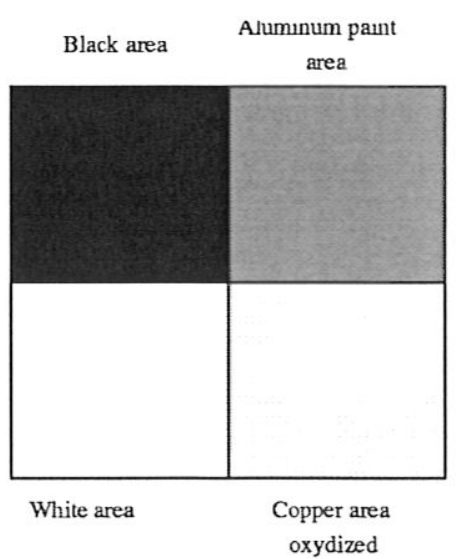

Fig. 27 Copper sheet.
Table 6 Experimental emissivity values.

\begin{tabular}{lcccc}
\hline \hline & Black Area & Aluminum Area & Copper Area & White Area \\
\hline$\varepsilon$ & $0.94 \pm 0.19$ & $0.67 \pm 0.15$ & $0.54 \pm 0.11$ & $0.48 \pm 0.12$ \\
\hline \hline
\end{tabular}

Both the VSU digital output value $I_{D_{i}}$ for each sheet area intensity $\left(L_{\mathrm{sp}_{1}}\right)$ and the $I_{D_{\mathrm{BB}}}$ value for a blackbody cavity intensity $\left(L_{s p_{0}}\right)$ are recorded at a distance of $1.5 \mathrm{~m}$ and with an integration time of $320 \mathrm{~ms}$. Finally, the four emissivities are computed directly with the relation $\varepsilon_{i}$ $=L_{\mathrm{sp}_{i}} / L_{\mathrm{sp}_{0}}=L_{D_{i}} / I_{D_{\mathrm{BB}}}$. The identified values are provided in Table 6 and are close to those given in the literature.

From Eq. (20) with the VSU digital output values $I_{D_{i}}$, the equivalent blackbody temperatures $T_{\text {eqi }}$ of each area are computed and compared to the reference copper temperature $T_{\text {ref }}$, as shown in Fig. 28. Next, the relative temperature uncertainty $\left[\Delta T / T=\left(T_{\text {ref }}-T_{\text {eqi }}\right) / T_{\text {ref }}\right]$ is derived, as illustrated in Fig. 29. An error of $57 \%$ in emissivity, resulting from the choice of a value 1 instead of 0.48 , leads to a relative temperature uncertainty $\Delta T / T$ of $7.2 \%$. Similarly, an error of $20 \%$ on emissivity only provides a relative temperature uncertainty $\Delta T / T$ of $2 \%$.

\subsubsection{Location of the source}

In the cargo area, the angle $\theta_{d}$ of the field of view varies between 0 and $40 \mathrm{deg}$. In this range of interest, the camera digital output $I_{D}$ decreases by only one gray level for a blackbody temperature of $425^{\circ} \mathrm{C}$ and at a minimal distance $d_{s}$ of $1.5 \mathrm{~m}$. The angle uncertainty infers a relative temperature uncertainty $\Delta T / T$ close to 0 .

Next, the source-camera distance influence is analyzed, although Eq. (4) does not depend on it if source focusing is assumed. The temperature uncertainty $\Delta T / T$ is shown in Fig. 30 for a source focused at a distance of $6 \mathrm{~m}$ directly viewing $\left(\theta_{d}=0 \mathrm{deg}\right)$ the blackbody at a temperature of $425^{\circ} \mathrm{C}$. Around this focused distance, no variation on the

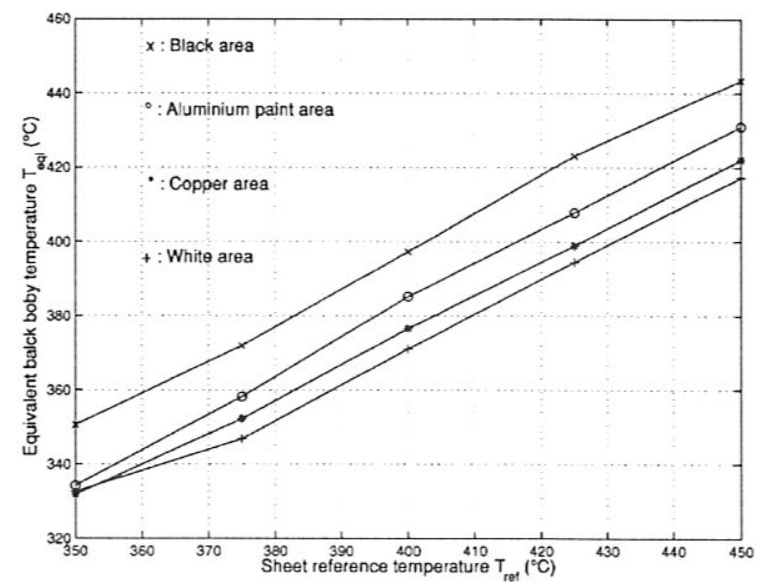

Fig. 28 Graph of $T_{\text {eqi }}$ versus $T_{\text {ref }}$. 


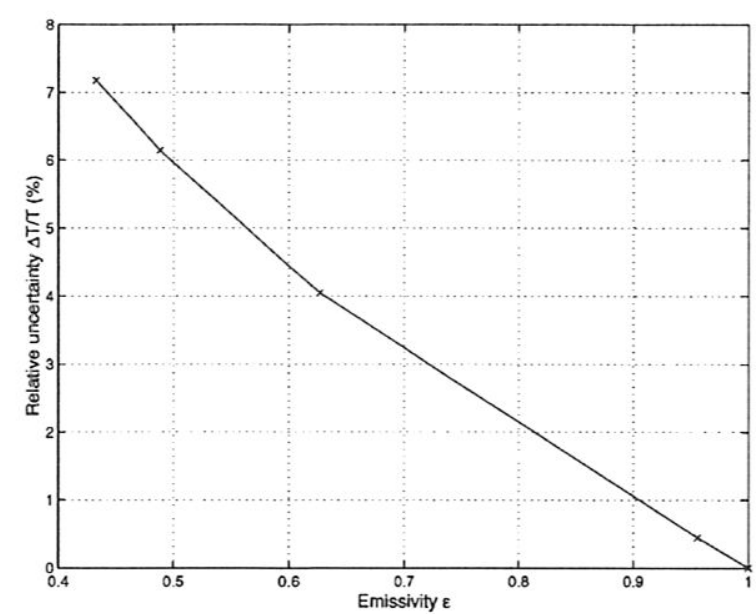

Fig. 29 Evolution of $\Delta T / T$ versus $\varepsilon$.

output signal $I_{D}$ is observed according to the assumption. Outside this distance and in the worst case, the maximal relative temperature uncertainty $\Delta T / T$ can be estimated to be as little as $2 \%$. The distance is limited to $10 \mathrm{~m}$ to consider that the hot source size in the image represents only a few pixels. In the next section, we describe a method for estimating the maximal total relative temperature uncertainty that takes into account the sum of the uncertainties.

\subsection{Temperature Uncertainties}

Theoretically, the maximal total relative temperature uncertainty $\Delta T / T$ can be expressed by the following relation:

$\frac{\Delta T}{T}=\left(\frac{\Delta T}{T}\right)_{i}+\left(\frac{\Delta T}{T}\right)_{s}$,

where $(\Delta T / T)_{i}$ is the instantaneous relative temperature uncertainty given by

$$
\left(\frac{\Delta T}{T}\right)_{i}=\frac{ \pm 3 \text { NETD }}{T} .
$$

From Eq. (21) and defining $A_{3}=A_{2} G_{D} t_{i}$, the static relative temperature uncertainty $(\Delta T / T)_{s}$ is given by

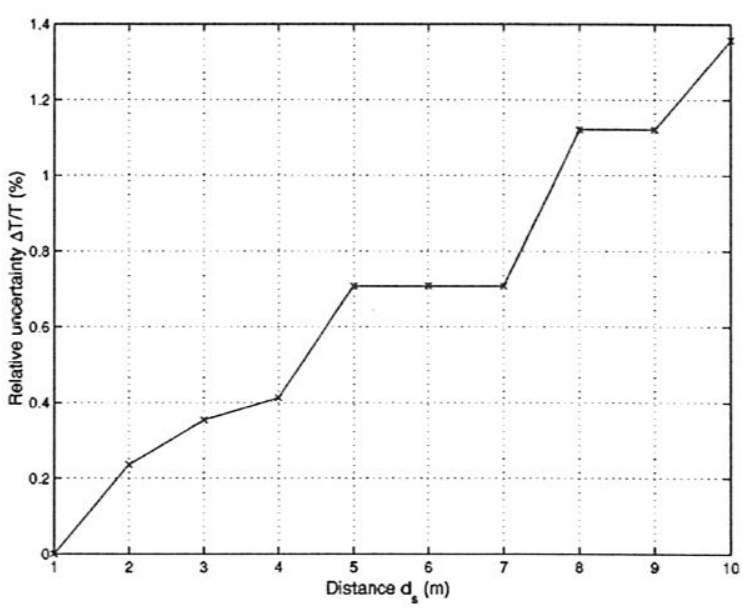

Fig. 30 Evolution of $\Delta T / T$ versus $d_{s}$

$$
\begin{aligned}
\left(\frac{\Delta T}{T}\right)_{s}= & \frac{\Delta B}{B}+\frac{f_{4}}{f_{6}\left(f_{4}+f_{5}\right)} \\
& \times\left[\frac{\Delta A_{3}}{A_{3}}+\frac{\Delta \varepsilon}{\varepsilon}+\frac{\Delta \tau_{e}}{\tau_{e}} \theta_{d} \tan \left(\theta_{d}\right) \frac{\Delta \theta_{d}}{\theta_{d}}\right. \\
& \left.+\frac{1}{f_{5}}\left(I_{D} \frac{\Delta I_{D}}{I_{D}}+C \frac{\Delta C}{C}\right)\right],
\end{aligned}
$$

where $f_{4}, f_{5}$, and $f_{6}$ are, respectively, equal to $A_{3} \tau_{e} \varepsilon \cos \left(\theta_{d}\right), I_{D}-C$, and $1 /\left[\ln \left(f_{4} / f_{5}+1\right)\right]$. The value of $\tau_{e}$ can be estimated by measuring the reflection coefficient of a known remote target illuminated by an emitting IR source. The associated relative uncertainty $\Delta \tau_{e} / \tau_{e}$ can be deduced from the camera output standard deviation $\left(\sigma_{I_{D}}\right)$. An output signal fluctuation $\Delta I_{D}$ greater than 6 times $\sigma_{I_{D}}$ should lead to a relative uncertainty of $10 \%$ for a distance of $15 \mathrm{~m}$. This point will be presented in a forthcoming paper. To take into account the lack of data in Eq. (24), we assume a transmittance value $\tau_{e}$ of only 1 (see the discussion in Sec. 2.2) with a relative uncertainty of $10 \%$. Moreover, the relative uncertainty of the $A_{3}, B, C$, and $I_{D}$ parameters represent the nonuniformity $\mathrm{CCD}$ array in the calibrated region. From the previous section, we assume that the relative angle uncertainty $\Delta \theta_{d} / \theta_{d}$ is close to zero.

For the parameter values summarized in Tables 2 and 7 , the static relative temperature uncertainty $(\Delta T / T)_{s}$ (see Fig. 31) stems mainly from the emissivity uncertainty (a minimum and a mean relative emissivity uncertainty are provided). For the same unfavorable configuration, the total

Table 7 Parameter values of Eq. (22).

\begin{tabular}{lcccccccc}
\hline \hline $\begin{array}{l}\Delta A_{3} / A_{3} \\
(\%)\end{array}$ & $\begin{array}{c}\Delta B / B \\
(\%)\end{array}$ & $\begin{array}{c}\Delta C / C \\
(\%)\end{array}$ & $\varepsilon$ & $\tau_{e}$ & $\begin{array}{c}\theta_{d} \\
(\mathrm{deg})\end{array}$ & $\begin{array}{c}\Delta \varepsilon / \varepsilon \\
(\%)\end{array}$ & $\begin{array}{c}\Delta \tau_{e} / \tau_{e} \\
(\%)\end{array}$ & $\begin{array}{c}t_{i} \\
(\mathrm{~ms})\end{array}$ \\
\hline 9.7 & 0.2 & 1.5 & 0.5 to 0.75 & 0.9 to 1 & 40 & 50 to 25 & 10 & 360 \\
\hline \hline
\end{tabular}




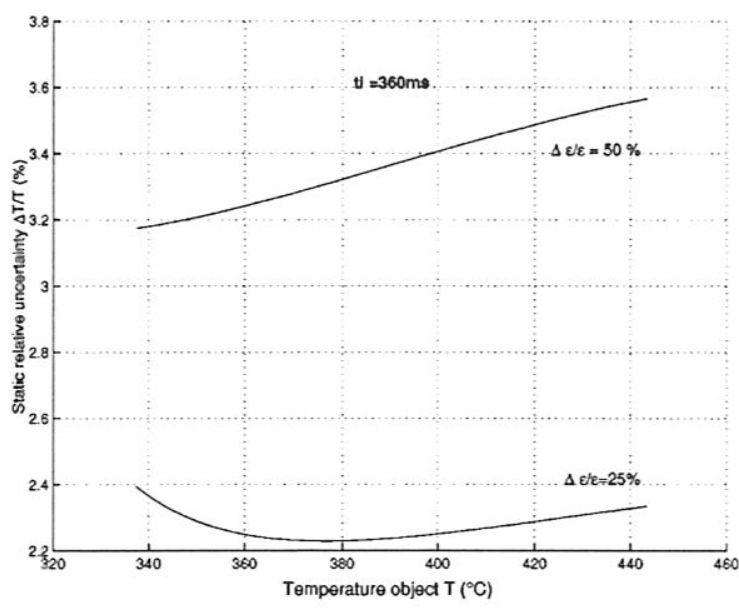

Fig. 31 Uncertainty $(\Delta T / T)_{s}$ versus $T$.

relative temperature uncertainty is plotted in Fig. 32. It depends mostly on the instantaneous relative temperature uncertainty that could be improved by using a higher integration time.

\subsection{Conclusion}

The maximal total relative temperature uncertainty is $5.5 \%$ in the worst measurement case and for a temperature of $350^{\circ} \mathrm{C}$. As a comparison, in Ref. 24 , a relative emissivity uncertainty $\Delta \varepsilon / \varepsilon$ of $20 \%$ leads to a relative temperature uncertainty $\Delta T / T$ of $1.2 \%$ at wavelength $\lambda_{e}=1 \mu \mathrm{m}$ and of $12 \%$ at $\lambda_{e}=10 \mu \mathrm{m}$. For short wavelengths, the emissivity is not as critical as in long wavelengths. The total absolute uncertainty $\Delta T$ is $\pm 16^{\circ} \mathrm{C}$ with a relative emissivity uncertainty of $\Delta \varepsilon / \varepsilon=25 \%$ (respectively, $\pm 20^{\circ} \mathrm{C}$ with $\Delta \varepsilon / \varepsilon$ $=50 \%$ ). These values fulfill the objective of a tolerance of $\pm 25^{\circ} \mathrm{C}$.

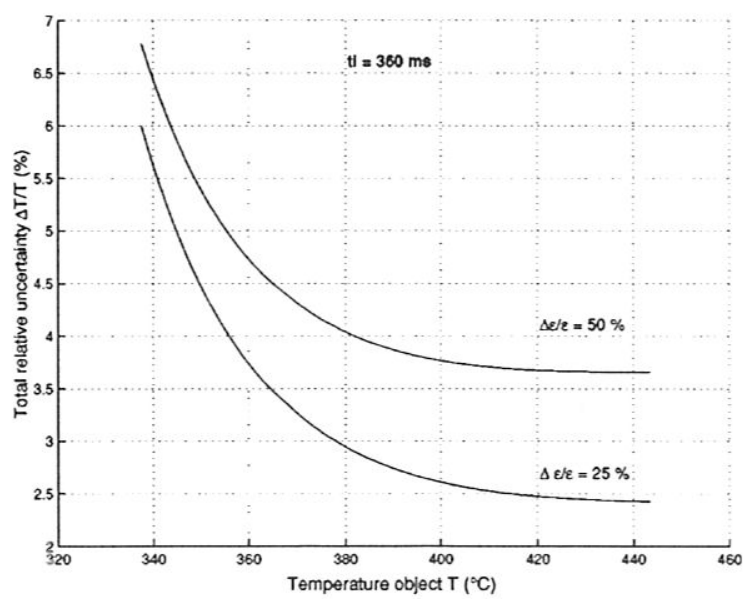

Fig. 32 Uncertainty $\Delta T / T$ versus $T$.

\section{Conclusions}

A new video sensor for multimeasurements (smoldering fires, flame, smoke, and load displacements) in aircraft cargo compartment is characterized. The new VSU, based on CCD technology, operates in the NIR spectral band $(0.75$ to $1.1 \mu \mathrm{m})$. The video sensor can also display the cargo area on a remote monitor to check the validity of the alarm signal. In this paper, the ability of the airborne sensor concept "one CCD technology/multimeasurements" was proved and a trade-off in sensor configuration was suggested. The radiometric and geometric models to measure, respectively, the fire temperature and the 3-D load localization were calibrated. The measurement of temperatures ranging from 350 to $900^{\circ} \mathrm{C}$ requires four exposure times from 360 to $0.1 \mathrm{~ms}$. The NETDs for this temperature range are less than $\pm 8^{\circ} \mathrm{C}$. In spite of the fact that the spatial band-limit frequency of a CCD camera decreases by a factor 1.5 in the NIR spectral band, the geometric model provides 3-D localization with an accuracy of $50 \mathrm{~mm}$ for an observation distance of $12.2 \mathrm{~m}$.

An analysis of the overheating conditions (emissivity and geometric situation) confirmed that working in shortwavelength $\mathrm{CCD}$ technology is less sensitive to a rough knowledge of the surface emissivity of an object. A relative uncertainty of emissivity $(\Delta \varepsilon / \varepsilon=50 \%)$ leads to a relative uncertainty of temperature $(\Delta T / T)$ lower than $5.5 \%$.

Airborne experiments revealed that other parameters might influence the measurements and in particular the cargo compartment temperature. Further investigations are currently in progress and focus on these aspects. The image analysis algorithms developed to perform smoldering fires, flame, smoke, and load displacement detections will be presented in a forthcoming paper.

\section{Appendix}

According to Ref. 25, the photon absorption coefficient is given for a photon energy $E$ between 1.2 and $2.2 \mathrm{eV}$ by (a)

$$
\begin{aligned}
\alpha= & 2750\left[\frac{E-1.1+5 \times 10^{-7} T_{d}^{2}}{\exp \left(348 / T_{d}\right)-1}\right. \\
& \left.+\frac{\left(E-1.16+5 \times 10^{-7} T_{d}^{2}\right)^{2}}{1-\exp \left(348 / T_{d}\right)}\right],
\end{aligned}
$$

and for a photon energy $E$ between 2.2 and $2.5 \mathrm{eV}$ by

$$
\alpha=\alpha\left(2.2 \mathrm{eV} ; T_{d}\right)\left[\frac{7447.3}{\alpha\left(2.2 \mathrm{eV} ; T_{d}\right)}+0.4491\right]^{(E / 0.3 \text { to } 7.3333)} \text {, }
$$

where $T_{d}$ is the detector temperature.

\section{Acknowledgments}

The authors wish to thank J. P. Arcens and B. David from École des Mines d'Albi-Carmaux, and C. Goin and G. Baudelet from the Latécoère group for their technical help. This research was supported by the Latécoère group. 


\section{References}

1. D. Blake, J. O'Sullivan, S. Hammann, M. Kolleck, and T. G. Cleary, "Aircraft cargo area fire detection," in Int. Aircraft Fire and Cabin Safety Research Conf., National Institute of Standards and Technology (1998)

2. J. A. Neal, C. E. Land, R. R. Avent, and R. J. Churchill, “Application of artificial neural networks to machine vision flame detection," Technical Report, American Research Corporation of Virginia, Radford (Apr. 1991)

3. A. Donald Goedeke, C. Fitzpatrick, and G. E. Healey, "Cost effective, dual purpose machine vision-based detectors for: (1) smoke and flame
detection, and (2) engine overheat/burn through and flame detection," Technical Report, Donmar LTD, Newport Beach (Mar. 1995).

4. Afnor, "S61-956-EN54-9. European requirements for industrial fire warning systems," Technical Report, Association française de normalisation (Afnor), Paris (Mar. 1997)

5. Afnor, "S61-954-EN547. European requirements for industrial fire warning systems," Technical Report, Association française de norwarning systems,' Technical

6. G. E. Healey and R. Kondepudy, "Radiometric CCD camera calibration and noise estimation," IEEE Trans. Pattern Anal. Mach. Intell. 16(3), 267 (Mar. 1994)

7. F. Moreau, "Thermographie proche infrarouge par caméras CCD et application aux composants de première paroi du Tokamak TORE SUPRA," PhD Thesis, Universite de Saint Jérôme-Aix-Marseille III (June 1996)

8. F. Mériaudeau, E. Renier, and F. Truchetet, "Uncertainty committed on temperature measurement," in Congrès International de Métrologie, Vol. 1, pp. 189-194, Nimes, France (Oct. 1995)

9. P. Saunders and T. Ricolfi, "The characterisation of a CCD camera for the purpose of temperature measurement" in TEMPMEKO'96-Int. Symp. on Temperature and Thermal Measurement in Industry and Science, pp. 329-334, Torino, Italy (1996).

10. Federal Aviation Administration, "TSO-C1C-technical standard order-cargo compartment fire detection instruments," Technical Reorder-cargo compartment fire detection instruments," Technical Report, Department of Transportation, Federal Aviation Administration

1. G. Boucourt, "Device for monitoring an enclosure, in particular the hold of an aircraft-patent PCT/FR99/00446," Technical Report, Latécoère, Toulouse, France (Jan. 1999)

12. G. C. Holst, CCD Arrays Cameras and Displays, SPIE Optical Engineering Press, Bellingham, WA (1998).

13. F. G. Smith, Ed., Atmospheric Propagation of Radiation, Vol. 2 of The Infrared \& Electro-Optical Systems Handbook, SPIE Press, Environmental Research Institute of Michigan, Ann Arbor (1992).

14. M. J. Modest, Ed., Radiative Heat Transfer, McGraw-Hill, New York (1993)

15. M. C. Dudzik, Ed., Electro-Optical Systems Design, Analysis, and Testing, Vol. 4 of The Infrared \& Electro-Optical Systems Handbook, SPIE Press, Environmental Research Institute of Michigan, Ann Arbor (1992).

16. D. H. Sieb, "Carrier diffusion degradation of modulation transfer function in charge coupled imagers," IEEE Trans. Electron Devices

17. D. Drysdale, Ed., An Introduction to Fire Dynamics, John Wiley and Sons, New York (1994)

18. T. Sentenac, J. J. Orteu, Y. Le Maoult, M. Devy, and G. Boucourt, "Load movement measurement using a near-infrared CCD camera for aircraft cargo surveillance," in Proc. 8th IEEE Int. Conf. on Emerging Technologies and Factory Automation, Antibes Juan-Les-Pins, France (Oct. 2001)

19. O. D. Faugeras, Three-Dimensional Computer Vision: A Geometric View Point, The MIT Press, Cambridge, MA (1993).

20. G. Weng, S. Ma, and M. Herniou, "Camera calibration with distortion models and accuracy evaluation," IEEE Trans. Pattern Anal. Mach. Intell. 14(10), 965-980 (1992).

21. H. A. Beyer, "Accurate calibration of CCD cameras," Proc. IEEE Conf. on Computer Vision and Pattern Recognition (CVPR'92), pp. 96-101. Champaign, IL (1992).

22. D. Garcia, J. J. Orteu, and M. Devy, "Accurate calibration of a stereovision sensor: comparison of different approaches," in Vision, reovision sensor: comparison of different approaches," in Vision, 2000). 2000).

23. F. Incropera and D. De Witt, Fundamentals of Heat and Mass Transfer, Wiley, New York (1985).

24. J. Martinet, La measure des températures par rayonnement thermique, Bureau National de Métrologie (1981)

25. G. Rolland, "Etude des variations de rendement quantique interne d'un détecteur CCD en fonction de la température," Rev. Phys. Appl. 20, 651-659 (Sep. 1985) 